Article

\title{
Synthesis of a Di-Mycoloyl Tri-Arabinofuranosyl Glycerol Fragment of the Mycobacterial Cell Wall, Based on Synthetic Mycolic Acids
}

\author{
Omar T. Ali ${ }^{1,+}\left(\mathbb{D}\right.$, Mohsin O. Mohammed ${ }^{1, \neq}{ }^{\circ}$, Juma'a R. Al Dulayymi ${ }^{2, *}$ and Mark S. Baird ${ }^{1, *}$ \\ 1 School of Chemistry, Bangor University, Bangor, Wales LL57 2UW, UK; omarkemia@yahoo.com.hk (O.T.A.); \\ althker1@yahoo.com (M.O.M.) \\ 2 School of Natural Sciences, Bangor University, Bangor, Wales LL57 2UW, UK \\ * Correspondence: j.aldulayymi@bangor.ac.uk (J.R.A.D.); chs028@bangor.ac.uk (M.S.B.) \\ + Current address: College of Education for Pure Science, Chemistry Department, Mosul University, \\ Mosul 41001, Iraq. \\ $\ddagger$ Current address: College of Science, Chemistry Department, Kirkuk University, Kirkuk, Iraq.
}

Academic Editors: Ramón J. Estévez Cabanas and Vito Ferro

Received: 12 August 2019; Accepted: 1 October 2019; Published: 6 October 2019

check for updates

\begin{abstract}
Fragments of mycobacterial cell walls such as arabinoglycerol mycolate and dimycoloyl diarabinoglycerol, comprising complex mixtures of mycolic acids, have immunostimulatory and antigenic properties. A related di-mycoloyl tri-arabinofuranosyl glycerol fragment has been isolated from cell wall hydrolysates. An effective stereoselective synthesis of tri-arabinofuranosyl glycerol, followed by coupling with stereochemically defined mycolic acids of different structural classes, to provide unique di-mycoloyl tri-arabinofuranosyl glycerols is now described.
\end{abstract}

Keywords: mycobacteria; cell membrane; mycolic acids; triarabinoglycerol

\section{Introduction}

The cell wall in Mycobacterium tuberculosis and in other mycobacteria has an unusual structure, containing a multi-layered and extremely hydrophobic envelope, which is important for the organism to survive in macrophages, and includes characteristic complex mixtures of long-chain (C70-C90), $\alpha$-alkyl branched $\beta$-hydroxylated fatty acids, 'mycolic acids' (MA); these include a range of groups (Figure 1), including cis- and trans-cyclopropanes, and cis- and trans-alkenes at positions $\mathrm{X}$ or $\mathrm{Y}$ and methoxy and keto-groups at position X [1,2]. The mycoloyl-arabinogalactan complex (mAG), the largest component structure in the cell wall of mycobacteria, is believed to act as a permeability barrier that prevents the passage of antibiotics. As well as being bound to the wall, largely as penta-arabinose tetramycolates, MA are also present as non-wall-bound sugar esters, such as trehalose dimycolate (TDM) and monomycolate (TMM), and as free acids. Hydrolysis of the cell wall of Mycobacterium bovis gave a penta-arabinose tetramycolate, and an arabinose mono-mycolate, as well as hexa-arabinose, hepta-arabinose and octa-arabinose tetramycolates [3]. Isolated natural arabinomycolates possess potent adjuvant immunostimulatory activity $[4,5]$. The preparation of a tetramycoloyl penta-arabinose using a complex natural mixture of MAs has been described [6-10]. Such fragments have also been found to be of value in the treatment of cancer [11]. Smaller fragments, such as glycerol mycolate [12-17] and arabinoglycerol mycolate $[18,19]$, have also been reported and, in the former case, have significant biological activity. In 1992, a new glycolipid, dimycoloyl diarabinoglycerol (DMAG) (2), was isolated from the Mycobacterium avium-Mycobacterium intracellulare complex (MAC) [20]. High immunoglobulin $\mathrm{M}$ (IgM) titres against the glycolipid were observed in enzyme-linked immunosorbent assays (ELISA) of serum from individuals who were culture positive for MAC infection, implying that this serodiagnosis 
detects the disease in an active phase [21]. A similar glycolipid mixture was isolated from M. bovis Bacille Calmette-Guérin or Mycobacterium marinum and from M. tuberculosis [22]. The DMAG from M. marinum was found to induce the secretion of proinflammatory cytokines (tumour necrosis factor $\alpha(\mathrm{TNF}-\alpha)$, and interleukins (IL-8, and IL-1 $\beta)$ ) in human macrophage THP-1 cells, to trigger the expression of the protein ICAM- 1 and cluster of differentiation 40 (CD40) cell surface antigens, and to modulate genes related to immune and inflammatory responses, suggesting that DMAG may drive host-pathogen interactions and participate in the immunopathogenesis of mycobacterial infections [23]. There is one report of the isolation of a dimycoloyl triarabinoglycerol (DMTAG, 3) from the degradation of the cell mycoloylarabinogalactan-peptidoglycan-protein complex from M. tuberculosis [24].

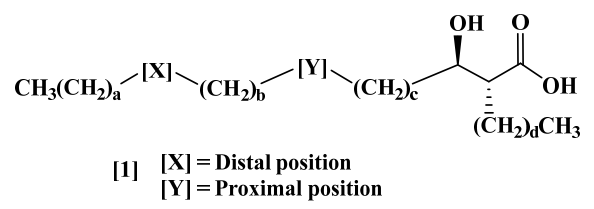

Figure 1. Generalised structure of mycolic acid
dimycoloyl triarabinoglycerol (DMTAG) (3).

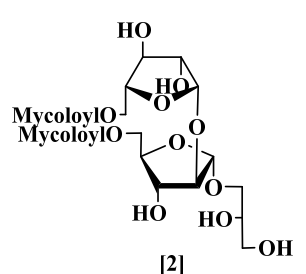

$[2]$

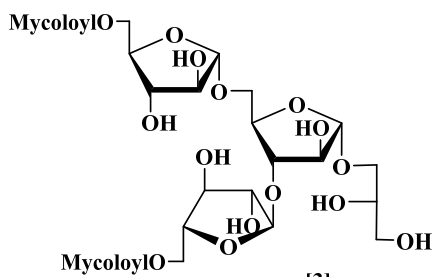

[3]

We have already reported the synthesis of triarabinose dimycolates [25], of arabinoglycerol mycolates [19], and of DMAGs containing unique synthetic mycolic acids [26]. Although, so far, it has no reported bioactivity, we now report the extension of the methods used in those synthetic approaches to the synthesis of a single stereoisomer of tri-arabinofuranosyl glycerol (TAG) and of a series of stereo-defined DMTAG glycolipids (Figure 1), through esterification of the glycan with structurally defined synthetic MAs of $\alpha$ - and keto-classes [1,2].

\section{Results and Discussion}

The target tri-saccharide structure (6) has three $\alpha$-glycosidic linkages and can be assembled readily from a donor (4) $[18,19]$ and a diol acceptor (5) (Scheme 1), a similar approach to the synthesis of the methoxy tri-saccharide of D-arabinofuranoside [25].
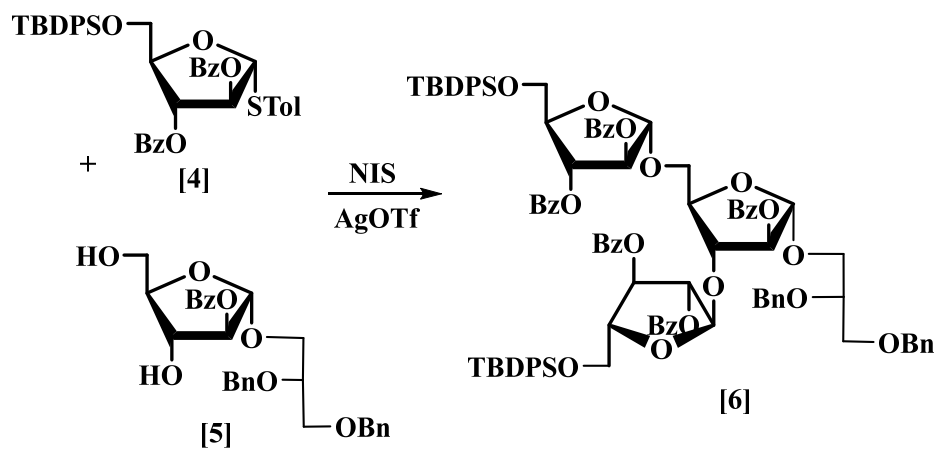

Scheme 1. Synthesis of fully protected glycan tri-arabino glycerol (TAG) (6).

The donor (4) was prepared before $[18,19]$. The target acceptor (5) was obtained in 95\% yield by desilylation of the protected arabinofuranosyl glycerol (7), prepared from D-arabinose [20], using tetrabutylammmonium fluoride in dry THF to give two free hydroxyl groups at the C-3 and C-5 positions, respectively (Scheme 2). 


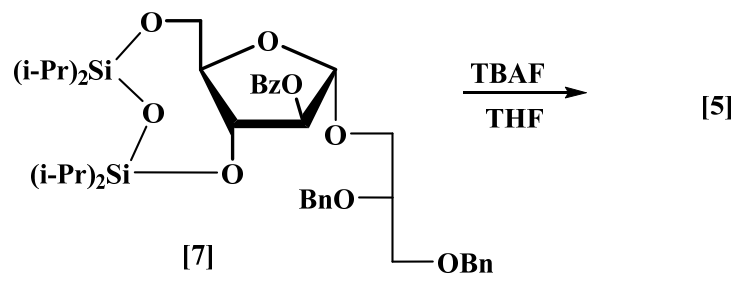

Scheme 2. Synthesis of the acceptor (5).

Following the method adopted by Liu et al. [6], the thioglycoside donor (4) was coupled with the acceptor (5) in the presence of N-iodosuccinimide (NIS)/silver trifluromethane sulfonate (AgOTf) in dry $\mathrm{CH}_{2} \mathrm{Cl}_{2}$ to give (6) in $91 \%$ yield. The ${ }^{1} \mathrm{H}-\mathrm{NMR}$ spectrum of (6) included three broad singlets at $\delta$ $5.61,5.31$ and 5.22, corresponding to the protons at the anomeric centre of each ring. The ${ }^{13} \mathrm{C}-\mathrm{NMR}$ spectrum (Figure S1, supporting information) established the presence of the $\alpha$-glycosidic linkages in the tri-arabinofuranosyl glycerol (6), with the signals at $\delta 106.1$ and $105.2 \mathrm{ppm}$ belonging to the three carbons at the anomeric centres. The HSQC-NMR of (6) (Figure S2), showed the three peaks corresponding to the acetal protons, at $\delta 5.61,5.31$ and 5.22, correlated to their carbons, in agreement with the assignments made by Liu et al. $[6,25]$.

The tri-saccharide (6) was debenzoylated with sodium methoxide to give (8) as a thick oil in $83 \%$ yield; in the ${ }^{1} \mathrm{H}-\mathrm{NMR}$ of (8), all the signals corresponding to the protons on the carbon adjacent to the benzoyl ester (6) were shifted up-field. The ${ }^{13} \mathrm{C}-\mathrm{NMR}$ spectrum showed the disappearance of the carbonyl signals. Compound (8) was benzylated to protect the five secondary hydroxyl groups using benzyl bromide (BnBr) and sodium hydride in dry dimethylformamide (DMF) in 65\% yield, followed by de-protection of the two primary hydroxyl groups using tetrabutylammonium fluoride (TBAF) to afford (9) in $87 \%$ yield (Scheme 3).
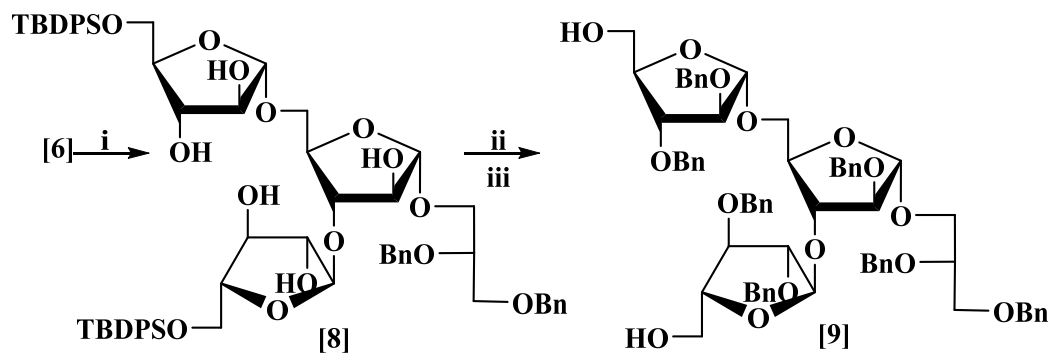

Scheme 3. Synthesis of compound (9). Reagents and conditions: (i) MeONa (1 M), then ethanoic acid $(\mathrm{AcOH}), 83 \%$; (ii) benzyl bromide $(\mathrm{BnBr}), \mathrm{NaH}$, dimethylformamide (DMF), 65\%; (iii) tetrabutylammonium fluoride (TBAF), tetrahydrofuran (THF), 87\%.

Compound (9) was then esterified, either by an alkylative coupling using cesium hydrogen carbonate after mesylation of the primary hydroxyl groups in the glycan or by direct coupling with a fatty acid using 1-ethyl-3-(3-dimethylaminopropyl)carbodi-imide (EDCI).

In the first method, the two primary hydroxyl groups in (9) were activated using methanesulfonyl chloride $(\mathrm{MsCl})$ in dry pyridine in the presence of catalytic 4-dimethylamino-pyridine (DMAP) in dry $\mathrm{CH}_{2} \mathrm{Cl}_{2}$ at $0{ }^{\circ} \mathrm{C}$ to afford the corresponding mesylate (10) in $87 \%$ yield (Scheme 4 ). The ${ }^{1} \mathrm{H}-\mathrm{NMR}$ spectrum of (10) (Figure S4) showed the expected signals, including two singlets at $\delta 2.94$ and 2.89 for the methyl groups of the mesylates. The ${ }^{13} \mathrm{C}$-NMR spectrum (Figure S5) showed two signals at $\delta 37.6$ and 37.5 for the carbons of the mesylates. The assignments of the signals were made by comparison with literature values reported for the methoxy tri-arabinose compound, which is identical to (10) except for the absence of the glycerol moiety $[16,17]$. 


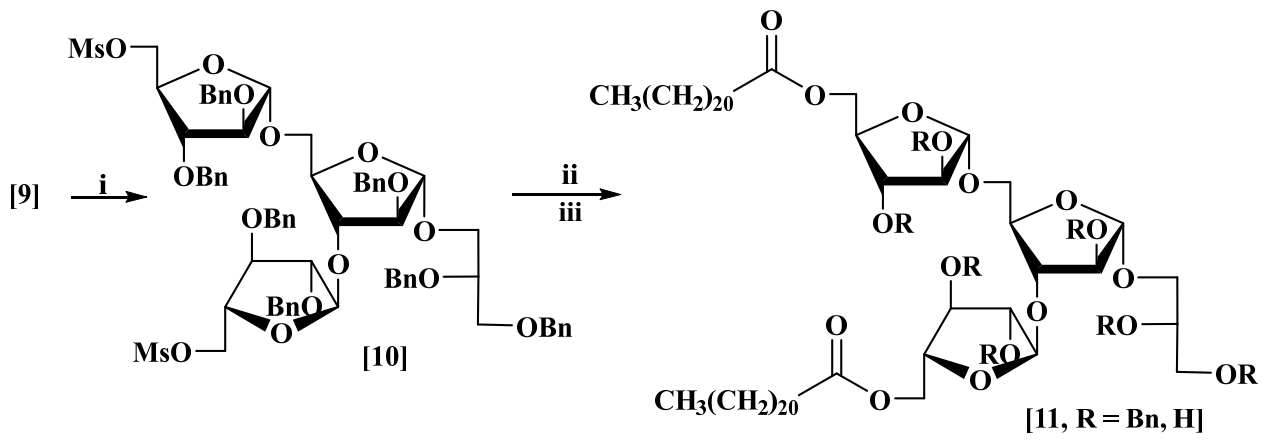

Scheme 4. Synthesis of a model di-behenoyl tri-arabinoglycerol $(\mathbf{1 1}, \mathrm{R}=\mathrm{H})$. Reagents and conditions: (i) methanesulfonyl chloride ( $\mathrm{MsCl}$ ), 4-dimethylaminopyridine (DMAP), pyridine, $87 \%$; (ii) $\mathrm{CsHCO}_{3}$, behenic acid, $\mathrm{DMF} / \mathrm{THF}, 70{ }^{\circ} \mathrm{C},(\mathbf{1 1}, \mathrm{R}=\mathrm{Bn}, 80 \%)$; (iii) $\mathrm{Pd}(\mathrm{OH})_{2} / \mathrm{C}(20 \%), \mathrm{H}_{2}, 82 \%$.

The protected di-behenoyl-triarabinoglycerol $(\mathbf{1 1}, \mathrm{R}=\mathrm{Bn})$ was prepared in $80 \%$ yield by coupling the mesylate (10) with behenic acid using cesium hydrogen carbonate in dry THF: DMF at $70{ }^{\circ} \mathrm{C}$ for 3 days (Scheme 4 ).

The ${ }^{1} \mathrm{H}-\mathrm{NMR}$ spectrum showed three broad one-proton singlets at $\delta 5.09,5.06$ and 4.97, corresponding to the three protons at the anomeric centres on the glycan rings. The $\mathrm{CH}_{2}$ groups adjacent to the carbonyls gave a triplet at $\delta 2.17(\mathrm{~J} 7.6 \mathrm{~Hz})$ integrating to four protons. The terminal methyl group showed a triplet signal at $\delta 0.81(\mathrm{~J} 6.8 \mathrm{~Hz})$ integrating to six protons. The ${ }^{13} \mathrm{C}-\mathrm{NMR}$ spectrum showed two signals at $\delta 173.6$ and 173.5 for the carbonyl groups. Signals corresponding to the carbon at the anomeric centre for the three rings appeared at $\delta 106.5,106.2$ and 105.5. The carbon of the $\mathrm{CH}_{2}$ group adjacent to the carbonyl in the acid appeared at $\delta 34.1$. The methylene chain ranged from $\delta 32-22$ and the terminal methyl group at $\delta 14.1$.

Compound $(11, \mathrm{R}=\mathrm{Bn})$ was debenzylated by stirring vigorously in a suspension of $\mathrm{Pd}(\mathrm{OH})_{2} / \mathrm{C}$ in dry $\mathrm{CH}_{2} \mathrm{Cl}_{2}$ :MeOH:THF (1:1:1.5) under an atmosphere of hydrogen for $36 \mathrm{~h}$ to give the target DMTAG analogue $(11, \mathrm{R}=\mathrm{H})$ in $82 \%$ yield (Scheme 4 ). The ${ }^{1} \mathrm{H}-\mathrm{NMR}$ spectrum (Figure S7) of compound $(\mathbf{1 1}, \mathrm{R}=\mathrm{H}$ ) showed three broad singlets at $\delta 5.01,4.97$ and $4.90 \mathrm{ppm}$ for the three $\alpha$-protons, with the remaining 20 protons of the sugar and glycerol moieties appearing between $\delta 4.30$ and $3.50 \mathrm{ppm}$. The four protons next to the two carbonyls gave a triplet at $\delta 2.30 \mathrm{ppm}(J 7.6 \mathrm{~Hz})$, while the terminal methyl groups appeared at $\delta 0.83 \mathrm{ppm}$ as a triplet $(J 6.5 \mathrm{~Hz})$. The ${ }^{13} \mathrm{C}-\mathrm{NMR}$ spectrum obtained for the glycolipid analogue $(\mathbf{1 1}, \mathrm{R}=\mathrm{H})$ gave signals (Figure S8), which were essentially identical to those for an analogue in the literature [25], bearing a methoxy substituent at C-1 rather than the glycerol substituent in compound $(\mathbf{1 1}, \mathrm{R}=\mathrm{H})$, and are assigned on that basis (See Supplementary Information, Table S1).

The effects of both free mycolic acids and of their sugar esters on immune responses, and on their recognition by disease antibodies, are known to depend on the specific classes they comprise, and on the detail of their stereochemistry [2,27-29]. The glycan (9) was therefore then coupled with three common classes of structurally defined synthetic MAs, cis-cyclopropane containing keto-MA (12a), $\alpha-\mathrm{MA}(\mathbf{1 2 b})$ and trans-cyclopropane containing keto-MA (12c) [30-32], followed by deprotection, as in Scheme 5 , to provide examples of three classes for the evaluation of their effects in these areas. In these cases, the coupling was achieved using EDCI-DMAP, which gave somewhat better yields based on the protected synthetic mycolic acids $[19,25]$. 


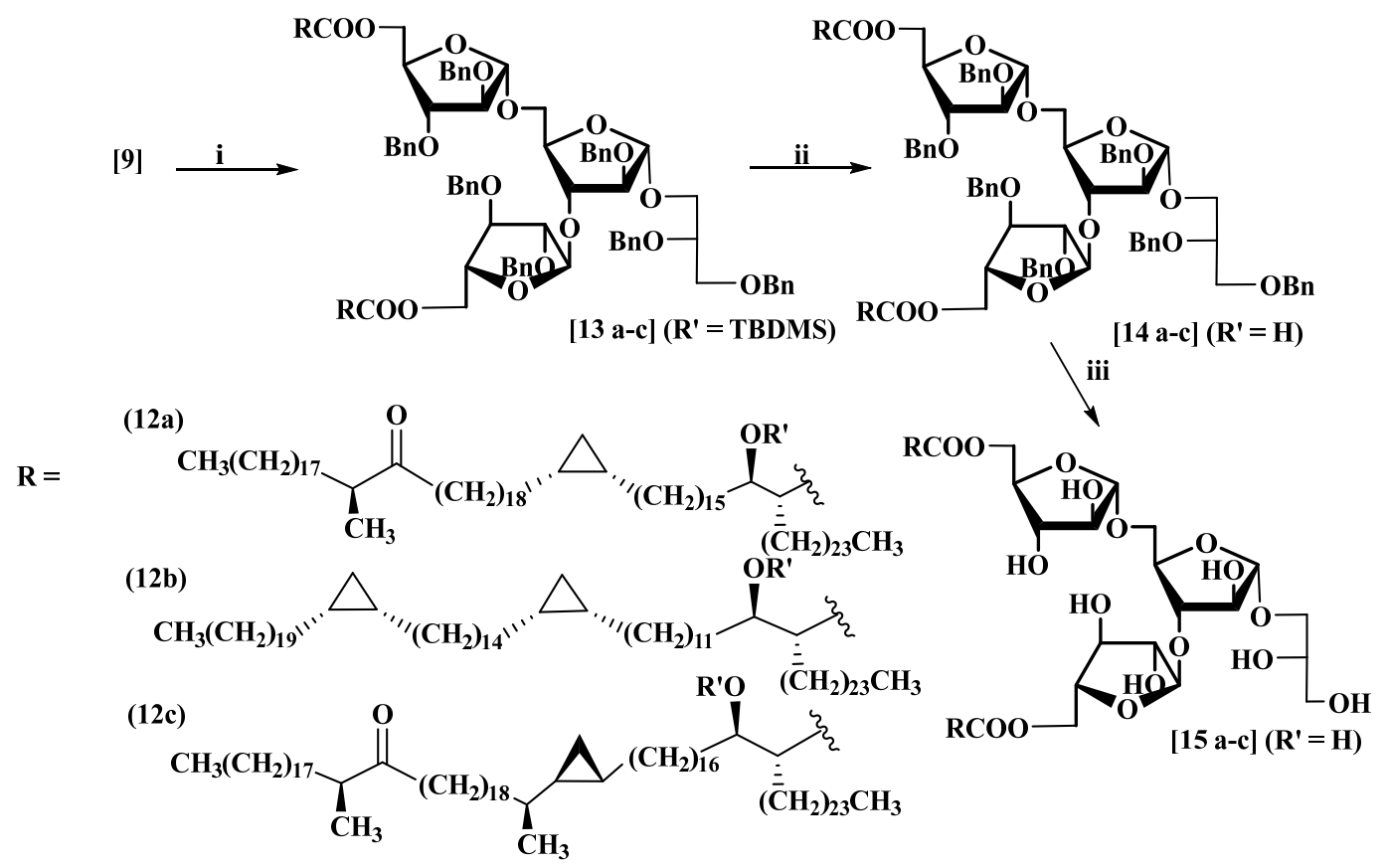

Scheme 5. Synthesis of dimycoloyl triarabinoglycerol (DMTAG) glycolipids. (i) RCOOH (R' = TBDMS) (12a-c), 1-ethyl-3-(3-dimethylaminopropyl)carbodi-imide (EDCI), DMAP, $\mathrm{CH}_{2} \mathrm{Cl}_{2}$ (a: 51\%; b: 84\%; c: $46 \%$ ); (ii) TBAF, THF (a 76\%; b: $51 \%$; c: $66 \%$ ); (iii) $\mathrm{Pd}(\mathrm{OH})_{2} / \mathrm{C}(20 \%), \mathrm{H}_{2}, \mathrm{CH}_{3} \mathrm{OH}: \mathrm{CH}_{2} \mathrm{Cl}_{2}$ : THF (1:1:1.5), rt, 36 h, (a: $86 \%$; b: $88 \%$; c: $82 \%)$.

The effects of the synthetic triarabinoglycerol mycolates in cytokine and chemokine stimulation, and their recognition by disease antibodies, are currently under investigation.

\section{Methods}

\subsection{General}

The chemicals used were obtained from commercial suppliers (Sigma-Aldrich, Lee on Solent, UK, and Alfa Aesar, Heysham, UK) or prepared from them by the methods described. Ether and tetrahydrofuran were dried over sodium wire and benzophenone under nitrogen, while dichloromethane was dried over calcium hydride. The petroleum spirit (petrol) used had boiling point of $40-60^{\circ} \mathrm{C}$. All reagents and solvents used were of reagent grade unless otherwise stated. Silica gel (Merck 7736) used for column and thin-layer chromatography was obtained from Sigma; separated components were detected variously using UV light, $\mathrm{I}_{2}$ and phosphomolybdic acid solution in IMS followed by charring. Anhydrous $\mathrm{MgSO}_{4}$ was used to dry organic solutions. Infrared (IR) spectra were carried out on a Perkin-Elmer 1600 F.T.I.R. spectrometer using liquid films or a KBr disc (solid). NMR spectra were carried out on Bruker Avance 400 or 500 spectrometers. $[\alpha]_{D}$ values were recorded in $\mathrm{CHCl}_{3}$ on a POLAAR 2001 optical activity polarimeter. Matrix-assisted laser desorption/ionization (MALDI) mass spectra were provided by the Engineering and Physical Science Research Council Mass Spectrometry Service in Swansea University.

\subsection{2', 3'-Di-O-benzyl-L-glycerol-(1' $\rightarrow 1)-2-O-b e n z o y l-\alpha$-D-arabinofuranoside (5)}

Tetrabutylammonium fluoride $(17.8 \mathrm{~mL}, 0.0616 \mathrm{~mol}, 1.0 \mathrm{M}$ in THF) was added dropwise with stirring to 2',3'-di-O-benzyl-L-glycerol-(1' $\rightarrow 1$ )-2-O-benzoyl-3,5-O-(tetraisopropylsiloxane-1,3-diyl)- $\alpha$-Darabinofuranoside (7) [20] $(6.7 \mathrm{~g}, 0.0089 \mathrm{~mol})$ in anhydrous THF $(50 \mathrm{~mL})$ at $0{ }^{\circ} \mathrm{C}$ under nitrogen. The mixture was allowed to reach room temperature $(\mathrm{rt})$ and was stirred for $4 \mathrm{~h}$, and it was then diluted with EtOAc $(100 \mathrm{~mL})$ and water $(50 \mathrm{~mL})$. The aqueous layer was re-extracted with EtOAc $(3 \times 50 \mathrm{~mL})$. The combined organic layers were washed with sat. aq. $\mathrm{NH}_{4} \mathrm{Cl}(50 \mathrm{~mL})$, brine $(50 \mathrm{~mL})$, 
dried and concentrated. Column chromatography eluting with petrol/EtOAc (1:1) gave compound (5) as a colourless thick oil $\left(4.3 \mathrm{~g}\right.$, 95\%) [MALDI: Found $(\mathrm{M}+\mathrm{Na})^{+}: 531.2 ; \mathrm{C}_{29} \mathrm{H}_{32} \mathrm{NaO}_{8}$ requires: 531.2], $[\alpha]_{D}^{23}+53\left(c 4.0, \mathrm{CHCl}_{3}\right)$, which showed $\delta_{\mathrm{H}}\left(400 \mathrm{MHz}, \mathrm{CDCl}_{3}\right): 7.98-7.93(2 \mathrm{H}, \mathrm{m}), 7.53(1 \mathrm{H}, \mathrm{t}, J 7.4 \mathrm{~Hz})$, $7.39(2 \mathrm{H}, \mathrm{t}, J 7.7 \mathrm{~Hz}), 7.33-7.17(10 \mathrm{H}, \mathrm{m}), 5.20(1 \mathrm{H}$, br. s), $5.04(1 \mathrm{H}$, br. s), $4.65(1 \mathrm{H}, \mathrm{d}, J 12.0 \mathrm{~Hz}), 4.61(1 \mathrm{H}$, d, J $12.0 \mathrm{~Hz}), 4.51(1 \mathrm{H}, \mathrm{d}, J 12.2 \mathrm{~Hz}), 4.48(1 \mathrm{H}, \mathrm{d}, J 12.2 \mathrm{~Hz}), 4.10(2 \mathrm{H}$, br. s), $3.85(1 \mathrm{H}, \mathrm{dd}, J 5.6,10.3 \mathrm{~Hz})$, $3.80(1 \mathrm{H}, \mathrm{m}), 3.76(1 \mathrm{H}$, br. p, J $5.2 \mathrm{~Hz}), 3.72-3.66(1 \mathrm{H}, \mathrm{m}), 3.63(1 \mathrm{H}, \mathrm{dd}, J 4.3,10.3 \mathrm{~Hz}), 3.60-3.52(2 \mathrm{H}$, including br. d, J 4.8 Hz at 3.58), 2.59-2.31 (2H, incl. $2 \times \mathrm{OH}$ groups); $\delta_{\mathrm{C}}\left(101 \mathrm{MHz}, \mathrm{CDCl}_{3}\right): 166.6$, 138.3, 138.0, 133.6, 129.8, 129.0, 128.5, 128.4, 128.3, 127.8, 127.6, 105.3, 85.9, 84.2, 76.6, 76.4, 73.4, 72.2, $69.7,67.1,61.9 ; v_{\max }: 3405,3065,3031,2945,2868,1715,1465,1105,884,712 \mathrm{~cm}^{-1}$.

\subsection{2', 3'-Di-O-benzyl-L-glycerol-( $\left(1^{\prime} \rightarrow 1\right)-2,3-d i-O-b e n z o y l-5-O-t e r t-b u t y l d i p h e n y l s i l y l-$ $\alpha$-D-arabinofuranosyl-(1 $\rightarrow 3)$-[2,3-di-O-benzoyl-5-O-tert-butyldiphenylsilyl- \\ $\alpha$-D-arabinofuranosyl-(1 $\rightarrow 5)]-2-O-b e n z o y l-\alpha$-D-arabinofuranoside (6)}

Molecular sieves $4 \AA$ (1.5 g) was added with stirring to furanoside (4) [18] (16.7 g, $28.9 \mathrm{mmol})$ and furanoside (5) $(4.2 \mathrm{~g}, 8.2 \mathrm{mmol})$ in dry $\mathrm{CH}_{2} \mathrm{Cl}_{2}(50 \mathrm{~mL})$ at rt under nitrogen. The mixture was stirred for $30 \mathrm{~min}$., then cooled to $-36^{\circ} \mathrm{C}$ and $\mathrm{N}$-iodosucinimide $(9.1 \mathrm{~g}, 0.037 \mathrm{~mol})$ was added followed by silver triflate $(2.1 \mathrm{~g}, 8.2 \mathrm{mmol})$. The mixture was stirred until it turned dark brown, quenched by the addition of triethylamine $(2 \mathrm{~mL})$ until it turned yellow, then diluted with $\mathrm{CH}_{2} \mathrm{Cl}_{2}(100 \mathrm{~mL})$ and filtered through celite. The filtrate was evaporated under reduced pressure; column chromatography eluting with hexane/EtOAc (5:2) gave compound (6) as a colourless thick oil (13.0 g, 91\%) [MALDI: Found $\left(\mathrm{M}+\mathrm{NH}_{4}\right)^{+}: 1682.6679 ; \mathrm{C}_{99} \mathrm{H}_{104} \mathrm{NO}_{20} \mathrm{Si}_{2}$ requires: 1682.6685] (Figure S3), $[\alpha]_{D}^{17}-1.4\left(c\right.$ 2.8, $\left.\mathrm{CHCl}_{3}\right)$; $\delta_{\mathrm{H}}\left(400 \mathrm{MHz}, \mathrm{CDCl}_{3}\right): 8.01-7.92(10 \mathrm{H}, \mathrm{m}), 7.70-7.67(4 \mathrm{H}, \mathrm{m}), 7.65-7.61(4 \mathrm{H}, \mathrm{m}), 7.58-7.53(2 \mathrm{H}, \mathrm{m})$, 7.50-7.44 (3H, m), 7.41-7.21 (32H, m), 5.66-5.62 (2H, incl. br. d, J $4.8 \mathrm{~Hz}$ at 5.63), $5.61(1 \mathrm{H}, \mathrm{br.s}), 5.55$ (1H, br.d, J 1.3 Hz), 5.51 (1H, br.d, J 0.9 Hz), 5.43 (1H, br.d, J 0.9 Hz), 5.31 (1H, br.s), $5.22(1 \mathrm{H}$, br.s), 4.68 $(2 \mathrm{H}$, br.s), $4.53(1 \mathrm{H}, \mathrm{d}, J 12.0 \mathrm{~Hz}), 4.49(1 \mathrm{H}, \mathrm{d}, J 12.0 \mathrm{~Hz}), 4.47(1 \mathrm{H}, \mathrm{br.s}), 4.40(1 \mathrm{H}, \mathrm{dd}, J 5.2,9.7 \mathrm{~Hz})$, 4.38-4.32 (2H, incl. br. dd, J 5.8, $10.5 \mathrm{~Hz}$ at 4.36), $4.04(1 \mathrm{H}, \mathrm{dd}, J 4.9,11.4 \mathrm{~Hz}), 3.95-3.89(5 \mathrm{H}, \mathrm{m}), 3.85$ $(1 \mathrm{H}, \mathrm{dd}, J 2.2,11.6 \mathrm{~Hz}), 3.81(1 \mathrm{H}, \mathrm{dd}, J 5.1,10.1 \mathrm{~Hz}), 3.68(1 \mathrm{H}, \mathrm{dd}, J$ 4.7, $10.4 \mathrm{~Hz}), 3.65-3.59(2 \mathrm{H}$, incl. br. t, J 4.7 Hz at 3.63), $1.00(9 \mathrm{H}, \mathrm{s}), 0.96(9 \mathrm{H}, \mathrm{s}) ; \delta_{\mathrm{C}}\left(101 \mathrm{MHz}, \mathrm{CDCl}_{3}\right): 165.5,165.4,165.2,165.1,138.6,138.3$, 135.7, 135.6, 135.5, 133.3, 133.2, 133.15, 133.1, 133.0, 130.0, 129.9, 129.8, 129.75, 129.7, 129.6, 129.35, 129.3, $129.25,129.2,128.4,128.35,128.3,128.25,128.2,127.8,127.7,127.6,127.55,127.5,127.4,126.3,106.1$, 105.2, 83.7, 83.4, 82.3, 82.2, 82.1, 81.8, 80.5, 77.2, 76.6, 73.3, 72.2, 70.1, 67.2, 66.1, 63.3, 26.7, 26.6, 19.3, 19.2; $v_{\max }: 3069,3010,2932,2857,1723,1602,1452,1072,706 \mathrm{~cm}^{-1}$.

3.4. $2^{\prime}, 3^{\prime}$-Di-O-benzyl-L-glycerol- $\left(1^{\prime} \rightarrow 1\right)-2,3-$ di-O-benzyl- $\alpha$-D-arabinofuranosyl- $(1 \rightarrow 3)$

[2,3-di-O-benzyl- $\alpha$-D-arabinofuranosyl-(1 $\rightarrow 5)]-2-O-b e n z y l-\alpha$-D-arabinofuranoside (9)

(a) Sodium methoxide ( $25 \mathrm{~mL}, 1 \mathrm{M}$, in methanol) was added to a stirred solution of furanoside (6) (9.1 g, $5.4 \mathrm{mmol})$ in dry $\mathrm{CH}_{2} \mathrm{Cl}_{2}: \mathrm{MeOH}(1: 1,50 \mathrm{~mL})$ at rt until a $\mathrm{pH}$ of 11 was obtained. The mixture was stirred for $90 \mathrm{~min}$, then neutralized by the addition of acetic acid. The solvent was evaporated under reduced pressure to give an oil; column chromatography eluting with chloroform/methanol (1:1) gave $2^{\prime}, 3^{\prime}$-di-O-benzyl-L-glycerol-( $\left.1^{\prime} \rightarrow 1\right)$-5-O-tert-butyldiphenyl-silyl- $\alpha$-D-arabino-furanosyl- $(1 \rightarrow 3)$ [5-O-tert-butyldiphenylsilyl- $\alpha$-D-arabinofuranosyl-( $1 \rightarrow 5)$ ]- $\alpha$-D-arabino-furanoside $(8)$ as a colourless thick oil $(5.1 \mathrm{~g}, 83 \%)$ [MALDI: Found $(\mathrm{M}+\mathrm{Na})^{+}: 1167.4913 ; \mathrm{C}_{64} \mathrm{H}_{80} \mathrm{NaO}_{15} \mathrm{Si}_{2}$ requires: 1167.4928], $[\alpha]_{D}^{17}+35\left(c\right.$ 6.7, $\left.\mathrm{CHCl}_{3}\right)$ which showed $\delta_{\mathrm{H}}\left(400 \mathrm{MHz}, \mathrm{CDCl}_{3}\right): 7.69-7.62(7 \mathrm{H}, \mathrm{m}), 7.51-7.18(23 \mathrm{H}, \mathrm{m})$, $5.18(1 \mathrm{H}$, br.s), $5.12(1 \mathrm{H}$, br.s), $4.93(1 \mathrm{H}$, br. s), $4.67(1 \mathrm{H}, \mathrm{d}, J 12.1 \mathrm{~Hz}), 4.63(1 \mathrm{H}, \mathrm{d}, J 12.1 \mathrm{~Hz}), 4.55(1 \mathrm{H}, \mathrm{d}, J$ $12.1 \mathrm{~Hz}), 4.51(1 \mathrm{H}, \mathrm{d}, J 12.1 \mathrm{~Hz}), 4.18(1 \mathrm{H}$, br. d, J $3.4 \mathrm{~Hz}), 4.16(1 \mathrm{H}$, br. dd, J 3.7, $6.1 \mathrm{~Hz}), 4.12(1 \mathrm{H}$, br. d, J $3.8 \mathrm{~Hz}), 4.07(2 \mathrm{H}$, br. s), 4.03-3.98 (2H, br. m), 3.98-3.93 (2H, incl. br. d, J $2.0 \mathrm{~Hz}$ at 3.96), $3.80(1 \mathrm{H}, \mathrm{dd}$, J 3.3, $8.8 \mathrm{~Hz}), 3.78-3.68(4 \mathrm{H}, \mathrm{m}), 3.67-3.64(1 \mathrm{H}, \mathrm{m}), 3.63-3.59(2 \mathrm{H}$, incl. br. dd, J 3.0, $8.1 \mathrm{~Hz}$ at 3.60), 3.57 $(1 \mathrm{H}, \mathrm{dd}, J 5.2,9.9 \mathrm{~Hz}), 3.02-2.55(7 \mathrm{H}, \mathrm{br} . \mathrm{m}), 1.05(9 \mathrm{H}, \mathrm{s}), 1.03(9 \mathrm{H}, \mathrm{s}) ; \delta_{\mathrm{C}}\left(101 \mathrm{MHz}, \mathrm{CDCl}_{3}\right): 138.4,138.2$, $135.6,135.5,131.9,131.8,131.7,131.6,130.2$, 130.1 130.0, 128.4, 128.35, 128.3, 128.0, 127.9, 127.8, 127.7, 
127.6, 127.55, 127.5, 108.6, 108.4, 108.3, 87.8, 87.4, 83.7, 82.3, 79.5, 78.8, 78.5, 77.8, 77.7, 76.7, 76.6, 73.3, 71.9, 69.7, 67.0, 66.0, 64.0, 63.8, 26.7, 26.6, 19.0, 18.9; $v_{\max }: 3418,3071,2933,2858,1454,1053,822 \mathrm{~cm}^{-1}$.

(b) A solution of the above $\alpha$-D-rabinofuranoside (8) $(5.0 \mathrm{~g}, 4.0 \mathrm{mmol})$ in dry DMF was added dropwise to a stirred suspension of $\mathrm{NaH}(1.0 \mathrm{~g}, 43 \mathrm{mmol})(60 \% \mathrm{w} / \mathrm{w}$, dispersion in mineral oil $)$ at $\mathrm{rt}$ under nitrogen. The mixture was stirred for $10 \mathrm{~min}$ then benzyl bromide $(5.2 \mathrm{~g}, 3.6 \mathrm{~mL}$, $30 \mathrm{mmol})$ in dry DMF $(2 \mathrm{~mL})$ was added, stirred at $\mathrm{rt}$ for $6 \mathrm{~h}$, then quenched by the slow addition of methanol $(2 \mathrm{~mL})$, and water $(10 \mathrm{~mL})$. The aqueous layer was re-extracted with EtOAc $(2 \times 50 \mathrm{~mL})$. The combined organic layers were washed with water $(25 \mathrm{~mL})$ and brine $(25 \mathrm{~mL})$, dried and evaporated under reduced pressure. Column chromatography eluting with petrol/EtOAc $(5: 1)$ gave $2^{\prime}, 3^{\prime}$-di-O-benzyl-L-glycerol-( $\left.1^{\prime} \rightarrow 1\right)$-2,3-di-O-benzyl-5-O-tert-butyldiphenylsilyl- $\alpha$-D-arabinofuranosyl-(1 $\rightarrow 3)$-[2,3-di-O-benzyl-5-O-tert-butyldiphenylsilyl- $\alpha$-D-arabinofuranosyl-(1 $\rightarrow 5)]-2-O-b e n z y l-\alpha-\mathrm{D}-$ arabinofuranoside as a colourless thick oil $(4.5 \mathrm{~g}, 65 \%)$ [MALDI: Found $(\mathrm{M}+\mathrm{Na})^{+}:$1617.7; $\mathrm{C}_{99} \mathrm{H}_{110} \mathrm{NaO}_{15} \mathrm{Si}_{2}$ requires: 1617.7], $[\alpha]_{D}^{17}+43\left(c 1.9, \mathrm{CHCl}_{3}\right)$ which showed $\delta_{\mathrm{H}}\left(400 \mathrm{MHz}, \mathrm{CDCl}_{3}\right)$ : 7.71-7.57 (8H, m), 7.48-7.13 (47H, m), $5.18(2 \mathrm{H}$, br. d, J $2.2 \mathrm{~Hz}), 5.07(1 \mathrm{H}$, br. s), $4.70(1 \mathrm{H}, \mathrm{d}, J 12.0 \mathrm{~Hz})$, $4.66(1 \mathrm{H}, \mathrm{d}, J 12.0 \mathrm{~Hz}), 4.58-4.50(7 \mathrm{H}, \mathrm{m}), 4.47(2 \mathrm{H}, \mathrm{d}, J 11.9 \mathrm{~Hz}), 4.46(1 \mathrm{H}, \mathrm{d}, J 11.9 \mathrm{~Hz}), 4.39(1 \mathrm{H}, \mathrm{d}, J$ $11.9 \mathrm{~Hz}), 4.38(1 \mathrm{H}, \mathrm{d}, J 11.9 \mathrm{~Hz}), 4.30(1 \mathrm{H}$, br.dd, J 2.8, $6.8 \mathrm{~Hz}), 4.18(2 \mathrm{H}$, br. m), 4.13-4.07 $(4 \mathrm{H}, \mathrm{m})$, 4.06-3.99 (2H, incl. br. dd, J 4.2, $10.6 \mathrm{~Hz}$ at 4.03), $3.95(1 \mathrm{H}, \mathrm{dd}, J 4.7,11.9 \mathrm{~Hz}), 3.87(1 \mathrm{H}, \mathrm{dd}, J 5.0,10.5$ $\mathrm{Hz}), 3.84-3.80(2 \mathrm{H}$, incl. br. dd, J 4.0, $11.0 \mathrm{~Hz}$ at 3.82), 3.79-3.74 $(4 \mathrm{H}, \mathrm{m}), 3.68-3.58(3 \mathrm{H}, \mathrm{m}), 1.03(18 \mathrm{H}, \mathrm{s})$; $\delta_{\mathrm{C}}\left(101 \mathrm{MHz}, \mathrm{CDCl}_{3}\right): 138.6,138.3,138.2,138.0,137.9,137.6,137.5,135.75,135.67,135.65,135.6,133.55$, $133.5,133.4,133.3,129.6,129.55,129.5,128.4,128.35,128.3,128.25,128.2,127.9,127.85,127.8,127.75$, $127.7,127.65,127.6,127.55,127.5,127.45,127.4,106.6,106.4,105.4,88.6,88.5,88.0,83.0,82.8,82.3,81.8$, $81.3,80.3,73.4,72.2,72.0,71.9,71.8,71.7,71.6,70.4,67.0,66.0,63.4,63.2,26.8,26.7,19.3,19.2 ; v_{\max }: 3067$, $3031,2929,2857,1495,1455,1111,698 \mathrm{~cm}^{-1}$.

(c) TBAF (14.3 mL, $0.0493 \mathrm{~mol}$, in $1.0 \mathrm{M}$ THF) was added dropwise to a stirred solution of the above furanoside $(3.8 \mathrm{~g}, 2.3 \mathrm{mmol})$ in anhydrous THF $(25 \mathrm{~mL})$ at $0{ }^{\circ} \mathrm{C}$ under nitrogen. The mixture was allowed to reach $\mathrm{rt}$ and stirred for $8 \mathrm{~h}$ then diluted with EtOAc $(100 \mathrm{~mL})$ washed with sat. aq. $\mathrm{NH}_{4} \mathrm{Cl}$ $(50 \mathrm{~mL})$ and brine $(50 \mathrm{~mL})$. The organic layer was dried and concentrated; column chromatography eluting with hexane/EtOAc (1:1) gave the title compound (9) as a colourless thick oil (2.3 g, 87\%) [MALDI-Found $(\mathrm{M}+\mathrm{Na})^{+}: 1141.5, \mathrm{C}_{67} \mathrm{H}_{74} \mathrm{NaO}_{15}$ requires: 1141.5$],[\alpha]_{D}^{17}+53\left(c\right.$ 4.7, $\left.\mathrm{CHCl}_{3}\right)$ which showed $\delta_{\mathrm{H}}\left(400 \mathrm{MHz}, \mathrm{CDCl}_{3}\right)$ : 7.34-7.16 $(35 \mathrm{H}, \mathrm{m}), 5.10(1 \mathrm{H}$, br. s), $5.07(1 \mathrm{H}$, br. s), $5.04(1 \mathrm{H}, \mathrm{br} . \mathrm{d}, J 0.7$ $\mathrm{Hz}), 4.64(2 \mathrm{H}$, br. s), $4.55-4.39(11 \mathrm{H}, \mathrm{m}), 4.31(1 \mathrm{H}, \mathrm{d}, J 11.7 \mathrm{~Hz}), 4.27(1 \mathrm{H}, \mathrm{dd}, J 3.8,7.4 \mathrm{~Hz}), 4.23-4.17$ $(1 \mathrm{H}, \mathrm{m}), 4.07(1 \mathrm{H}$, br. d, J $2.2 \mathrm{~Hz}), 4.06(1 \mathrm{H}$, br. dd, J 4.0, $7.6 \mathrm{~Hz}), 4.02(1 \mathrm{H}$, br. dd, J 2.2, $5.9 \mathrm{~Hz}), 3.98(1 \mathrm{H}$, br. dd, J 1.3, $3.8 \mathrm{~Hz}), 3.96(1 \mathrm{H}$, br. dd, $J 1.2,3.6 \mathrm{~Hz}), 3.88(1 \mathrm{H}$, br. dd, $J 4.1,12.3 \mathrm{~Hz}), 3.85(1 \mathrm{H}$, br. dd, $J$ 3.2, $6.5 \mathrm{~Hz}), 3.82(1 \mathrm{H}, \mathrm{d}, J 5.2 \mathrm{~Hz}), 3.78(1 \mathrm{H}, \mathrm{dd}, J 3.7,7.4 \mathrm{~Hz}), 3.76-3.72(2 \mathrm{H}, \mathrm{m}), 3.71-3.64(2 \mathrm{H}$, incl. br. dd, $J 12.3,2.4 \mathrm{~Hz}$ at 3.68$), 3.61(1 \mathrm{H}, \mathrm{dd}, J 4.8,7.3 \mathrm{~Hz}), 3.59-3.55(3 \mathrm{H}$, incl. br. $\mathrm{d}, J 5.1 \mathrm{~Hz}$ at 3.58$), 3.53$ $(1 \mathrm{H}, \mathrm{dd}, J 5.9,12.3 \mathrm{~Hz}), 1.5\left(2 \mathrm{H}, \mathrm{br}\right.$ s); $\delta_{\mathrm{C}}\left(101 \mathrm{MHz}, \mathrm{CDCl}_{3}\right): 138.5,138.2,137.7,137.6,137.5,137.4,137.2$, 128.5, 128.45, 128.4, 128.35, 128.3, 128.0, 127.95, 127.9, 127.85, 127.8, 127.75, 127.7, 127.6, 127.5, 106.1, 106.0, 105.9, 88.7, 88.3, 87.4, 83.0, 82.9, 82.4, 81.9, 80.8, 79.8, 73.4, 72.3, 72.25, 72.2, 72.0, 71.9, 71.8, 70.2, $67.2,64.8,62.7 ; v_{\max }: 3459,3064,3030,2921,2860,1605,1496,1115,820 \mathrm{~cm}^{-1}$.

3.5. 2', $3^{\prime}$-Di-O-benzyl-L-glycerol-(1' $\left.\rightarrow 1\right)-2,3-d i-O-b e n z y l-5-O-m e t h a n e s u l f o n y l-\alpha-\mathrm{D}-$ arabinofuranosyl-(1 $\rightarrow 3)-[2,3-$ di-O-benzyl-5-O-methanesulfonyl- $\alpha$-Darabinofuranosyl- $(1 \rightarrow 5)]-2-O-b e n z y l-\alpha$-D-arabinofuranoside $(\mathbf{1 0})$

Methanesulfonyl chloride $(0.57 \mathrm{~g}, 0.40 \mathrm{~mL}, 0.0050 \mathrm{~mol})$ and DMAP $(0.05 \mathrm{~g}, 0.43 \mathrm{mmol})$ were added to a stirred solution of furanoside $(9)(0.56 \mathrm{~g}, 0.50 \mathrm{mmol})$ in dry pyridine $(5 \mathrm{~mL})$ under nitrogen at $\mathrm{rt}$. After $16 \mathrm{~h}$ it was quenched by the addition of water $(1 \mathrm{~mL})$; the organic layer was diluted with $\mathrm{CH}_{2} \mathrm{Cl}_{2}$ $(10 \mathrm{~mL})$ then washed with $1 \mathrm{M}$ aq. $\mathrm{HCl}(2 \times 10 \mathrm{~mL})$, sat. aq. $\mathrm{NaHCO}_{3}(2 \times 10 \mathrm{~mL})$, dried and evaporated to give a thick oil; column chromatography eluting with petrol/EtOAc (3:1) gave the title compound (10) as a colourless thick oil $(0.55 \mathrm{~g}, 87 \%)$ [MALDI: Found $(\mathrm{M}+\mathrm{Na})^{+}: 1297.4 ; \mathrm{C}_{69} \mathrm{H}_{78} \mathrm{NaO}_{19} \mathrm{~S}_{2}$ requires: 1297.4], $[\alpha]_{D}^{17}+59\left(\right.$ c 0.60, $\left.\mathrm{CHCl}_{3}\right)$, which showed $\delta_{\mathrm{H}}\left(400 \mathrm{MHz}, \mathrm{CDCl}_{3}\right): 7.40-7.15(35 \mathrm{H}, \mathrm{m}), 5.16(1 \mathrm{H}, \mathrm{br}$. 
s), $5.13(1 \mathrm{H}$, br. s), 5.07 (1H, br. s), 4.68 (2H, br. s), 4.57-4.43 (11H, m), $4.40(1 \mathrm{H}, \mathrm{d}, \mathrm{J} 11.3 \mathrm{~Hz}), 4.38-4.34$ $(1 \mathrm{H}, \mathrm{m}), 4.33(1 \mathrm{H}$, br.dd, J 3.9, $7.3 \mathrm{~Hz}), 4.30-4.26(2 \mathrm{H}$, incl. br. dd, J 3.4, $7.3 \mathrm{~Hz}$ at 4.29), $4.24(1 \mathrm{H}, \mathrm{d}, J 5.4$ $\mathrm{Hz}), 4.22-4.12(2 \mathrm{H}, \mathrm{m}), 4.11-4.07(2 \mathrm{H}, \mathrm{m}), 4.02-3.97(2 \mathrm{H}, \mathrm{m}), 3.91-3.84(4 \mathrm{H}, \mathrm{m}), 3.80(1 \mathrm{H}, \mathrm{br} . \mathrm{p}, \mathrm{J} 4.8 \mathrm{~Hz})$, $3.73(1 \mathrm{H}$, br. dd, $J 1.5,11.5 \mathrm{~Hz}), 3.67-3.59$ (3H, incl. br. dd, J 4.8, 8.7 Hz at 3.64), $2.94(3 \mathrm{H}, \mathrm{s}), 2.89$ (3H, s); $\delta_{\mathrm{C}}\left(101 \mathrm{MHz} \mathrm{CDCl}_{3}\right): 138.5,138.2,137.4,137.3,137.2,137.0,128.6,128.55,128.5,128.45,128.4,128.3$, $128.1,128.05,128.0,127.95,127.9,127.7,127.65,127.6,127.55,106.5,106.1,105.8,88.3,87.7,87.5,82.9$, $82.8,80.3,80.2,79.3,79.2,77.2,73.4,72.35,72.3,72.2,72.1,72.0,71.9,70.2,68.8,68.5,67.2,65.2,37.6,37.5$; $v_{\max }: 3088,3065,3031,2933,2871,1586,1454,1177,745 \mathrm{~cm}^{-1}$.

3.6. L-Glycerol-( $\left(1^{\prime} \rightarrow 1\right)-5-O-b e h e n a t e-\alpha$-D-arabinofuranosyl- $(1 \rightarrow 3)-5-O-b e h e n a t e-\alpha$-Darabinofuranosyl- $(1 \rightarrow 5)-\alpha$-D-arabinofuranoside $(\mathbf{1 1}, R=H)$

(a) $\mathrm{CsHCO}_{3}(0.076 \mathrm{~g}, 0.39 \mathrm{mmol})$ was added to a stirred solution of dimesylate (10) (0.05 g, $0.03 \mathrm{mmol})$ and behenic acid $(0.03 \mathrm{~g}, 0.09 \mathrm{mmol})$ in dry DMF:THF $(1: 5,1 \mathrm{~mL})$ at rt under nitrogen. The mixture was stirred at $70{ }^{\circ} \mathrm{C}$ for 3 days, then diluted with EtOAc (25 mL) and water $(5 \mathrm{~mL})$. The aqueous layer was re-extracted with EtOAc $(2 \times 10 \mathrm{~mL})$. The combined organic layers were washed with water $(5 \mathrm{~mL})$ and brine $(5 \mathrm{~mL})$, dried and evaporated under reduced pressure to give a thick oil; column chromatography eluting with petrol/EtOAc (3:1) gave $2^{\prime}, 3^{\prime}$-di-O-benzyl-L-glycerol-( $\left(1^{\prime} \rightarrow 1\right)$-2,3-di-O-benzyl-5-O-behenate- $\alpha$-D-arabinofuranosyl- $(1 \rightarrow 3)$ [2,3-di-O-benzyl-5-O-behenate- $\alpha$-D-arabinofuranosyl-(1 $\rightarrow 5)]-2-O-b e n z y l-\alpha$-D-arabinofuranoside (11, $\mathrm{R}=\mathrm{Bn})$ as a colourless thick oil $(55 \mathrm{mg}, 80 \%)$ [MALDI: Found $(\mathrm{M}+\mathrm{Na})^{+}: 1786.1 ; \mathrm{C}_{111} \mathrm{H}_{158} \mathrm{NaO}_{17}$ requires: 1786.1$],[\alpha]_{D}^{22}+36\left(c 1.0, \mathrm{CHCl}_{3}\right)$ which showed $\delta_{\mathrm{H}}\left(400 \mathrm{MHz}, \mathrm{CDCl}_{3}\right): 7.31-7.11(35 \mathrm{H}, \mathrm{m})$, 5.09 (1H, br. s), 5.06 (1H, br. s), 4.97 (1H, br. s), 4.59 (2H, br. s), 4.51-4.35 (10H, m), $4.33(1 \mathrm{H}, \mathrm{d}, \mathrm{J} 11.8$ Hz), $4.26(1 \mathrm{H}, \mathrm{d}, J 11.8 \mathrm{~Hz}), 4.20(1 \mathrm{H}$, br. dd, J 3.3, $7.3 \mathrm{~Hz}), 4.12(6 \mathrm{H}$, br. m), $4.04(1 \mathrm{H}$, br. dd, J 2.8, 6.9 Hz), 4.00 (1H, br. d, J $2.5 \mathrm{~Hz}), 3.95-3.89$ (2H, m), $3.84(1 \mathrm{H}, \mathrm{dd}, J 4.3,11.8 \mathrm{~Hz}), 3.81-3.76(2 \mathrm{H}$, incl. br. dd, J 4.1, 8.5 Hz at 3.78), $3.75(1 \mathrm{H}$, br. d, J 3.4 Hz), 3.74-3.68 (1H, p, J 5.0 Hz), $3.66(1 \mathrm{H}$, br. dd, J 2.1, 11.7 Hz), 3.58-3.49 (3H, incl. br. q, J 4.6 Hz at 3.53), $2.17(4 \mathrm{H}, \mathrm{t}, J 7.6 \mathrm{~Hz}), 1.56-1.00(76 \mathrm{H}, \mathrm{m}), 0.81(6 \mathrm{H}, \mathrm{t}, \mathrm{J} 6.8 \mathrm{~Hz})$; $\delta_{\mathrm{C}}\left(101 \mathrm{MHz} \mathrm{CDCl}_{3}\right): 173.6,173.5,138.6,138.3,137.7,137.6,137.5,137.4,137.3,128.5,128.4,128.35$, $128.3,128.0,127.95,127.9,127.85,127.8,127.75,127.7,127.65,127.6,127.5,106.5,106.2,105.5,88.3,88.2$, 88.1, 83.4, 83.3, 80.8, 80.3, 79.2, 78.9, 76.9, 73.4, 72.3, 72.2, 72.1, 72.0, 71.9, 71.7, 70.3, 67.1, 65.6, 63.3, 63.2, $34.1,34.0,31.9,29.7,29.6,29.5,29.4,29.35,29.3,29.2,24.8,22.7,14.1 ; v_{\max }: 3064,3031,2923,2853,1740$, $1718,1455,1066,698 \mathrm{~cm}^{-1}$.

(b) A general procedure for debenzylation was used throughout: Palladium hydroxide on activated charcoal $\left(\mathrm{Pd}(\mathrm{OH})_{2}-\mathrm{C} / 20 \%\right.$ (1.1 fold by weight) was added to a stirred solution of the benzyl protected compound $(0.010 \mathrm{mmol})$ in $\mathrm{CH}_{2} \mathrm{Cl}_{2}$ :MeOH:THF $(1: 1: 1.5,2 \mathrm{~mL})$ at rt under hydrogen. The mixture was stirred for $36 \mathrm{~h}$ then filtered through celite and the solvent was evaporated under reduced pressure to give a residue; column chromatography eluting with chloroform/methanol (10:1) afforded the desired compound. In this way, compound $(\mathbf{1 1}, \mathrm{R}=\mathrm{H})$ was obtained as a colourless thick oil $(29 \mathrm{mg}, 82 \%)$ [MALDI: Found $(\mathrm{M}+\mathrm{Na})^{+}: 1155.8 ; \mathrm{C}_{62} \mathrm{H}_{116} \mathrm{NaO}_{17}$ requires: 1155.8$],[\alpha]_{D}^{18}-21\left(c 1.1, \mathrm{CHCl}_{3}\right)$, which showed $\delta_{\mathrm{H}}\left(400 \mathrm{MHz}, \mathrm{CDCl}_{3}+\right.$ few drops $\left.\mathrm{CD}_{3} \mathrm{OD}\right): 5.01(1 \mathrm{H}$, br. s), $4.97(1 \mathrm{H}, \mathrm{br} . \mathrm{s}), 4.90(1 \mathrm{H}, \mathrm{br} . \mathrm{s})$, 4.28-4.20 (2H, incl. br. dd J 3.2, $11.7 \mathrm{~Hz}$ at 4.24), 4.19-4.13 (3H, incl. br. dd J 5.0, $11.8 \mathrm{~Hz}$ at 4.17), 4.12 $(1 \mathrm{H}$, br. d, J $4.3 \mathrm{~Hz}), 4.11-4.08(1 \mathrm{H}, \mathrm{m}), 4.04(1 \mathrm{H}$, br. q, J $5.5 \mathrm{~Hz}), 4.01-3.95(3 \mathrm{H}, \mathrm{br} . \mathrm{m}), 3.94(1 \mathrm{H}, \mathrm{dd}, J$ 3.6, $11.5 \mathrm{~Hz}), 3.83-3.76(3 \mathrm{H}, \mathrm{m}), 3.73(1 \mathrm{H}, \mathrm{br} . \mathrm{dd}, \mathrm{J} 4.8,10.1 \mathrm{~Hz}), 3.67-3.62(1 \mathrm{H}, \mathrm{m}), 3.62-3.57(2 \mathrm{H}$, incl. br. d, J 3.1 Hz at 3.60), 3.56-3.51 (1H, m), $2.30(4 \mathrm{H}, \mathrm{t}, J 7.6 \mathrm{~Hz}), 1.64-1.02(83 \mathrm{H}, \mathrm{m}), 0.83(6 \mathrm{H}, \mathrm{t}, \mathrm{J} 6.5 \mathrm{~Hz})$; $\delta_{\mathrm{C}}\left(101 \mathrm{MHz} \mathrm{CDCl}_{3}\right): 174.05,174.0,108.0,107.7,83.3,83.0,82.4,81.8,81.3,80.7,79.0,77.6,75.8,69.9$, $69.1,66.4,63.9,63.7,63.5,34.0,33.95,31.8,29.6,29.5,29.45,29.4,29.3,29.25,29.2,29.1,29.0,24.8,24.7$, 22.6, 13.9.; $v_{\max }: 3374,2920,2852,1730,1723,1180,757 \mathrm{~cm}^{-1}$. 
3.7. 12L-Glycerol-(1' $\rightarrow 1)-5-O-(2 R)-2-(1-h y d r o x y-16-((1 S, 2 R)-2-((S)-20-m e t h y l-19-$

oxooctatria-contyl)cyclopropyl)hexadecyl)hexacosanoate- $\alpha$-D-arabinofuranosyl-(1 $\rightarrow 3)-[5-O-((2 R)-2-(1-h y d r o x y-$ 16-((1S,2R)-2-((S)-20-methyl-19-oxooctatriacontyl)cyclopropyl)hexadecyl)hexacosan-oate- $\alpha$-D-arabinofuranosyl$(1 \rightarrow 5)]-\alpha$-D-arabinofuranoside (15a)

(a) 1-Ethyl-3-(3-dimethylaminopropyl)carbodiimide hydrochloride (EDCI) (60 $\mathrm{mg}$, $0.31 \mathrm{mmol})$, in dry $\mathrm{CH}_{2} \mathrm{Cl}_{2}(1 \mathrm{~mL})$ was added to a stirred solution of $\alpha$-D-arabinofuranoside (9) $(35 \mathrm{mg}, 0.031 \mathrm{mmol})$, molecular sieves $4 \AA$ (50 mg), DMAP (38 mg, 0.31) (2R)-2-(1-((tert-butyldimethyl-silyl)oxy)-16-((1S,2R)-2-((S)-20-methyl-19-oxooctatriacontyl)cyclopropyl) hexadecyl)hexacosanoic acid (12a) $(85 \mathrm{mg}, 0.062 \mathrm{mmol})$ [30] in dry $\mathrm{CH}_{2} \mathrm{Cl}_{2}(1 \mathrm{~mL})$ at rt under nitrogen. After 4 days, the precipitate was filtered off and washed with $\mathrm{CH}_{2} \mathrm{Cl}_{2}(10 \mathrm{~mL})$, and the solvent was evaporated; column chromatography eluting with hexane/EtOAc (10:1) gave compound (13a) as a colourless thick oil $(60 \mathrm{mg}, 51 \%)$ [MALDI: Found $(\mathrm{M}+\mathrm{Na})^{+}: 3808.2 ; \mathrm{C}_{247} \mathrm{H}_{426} \mathrm{NaO}_{21} \mathrm{Si}_{2}$ requires: 3808.2], $[\alpha]_{D}^{20}+18\left(c 5.0, \mathrm{CHCl}_{3}\right)$, which showed $\delta_{\mathrm{H}}\left(400 \mathrm{MHz}, \mathrm{CDCl}_{3}\right): 7.69-6.95(35 \mathrm{H}, \mathrm{m}), 5.16(1 \mathrm{H}, \mathrm{br}$. s), $5.13(1 \mathrm{H}$, br. s), $5.04(1 \mathrm{H}$, br. s), $4.68(1 \mathrm{H}, \mathrm{d}, J 12.1 \mathrm{~Hz}), 4.65(1 \mathrm{H}, \mathrm{d}, J 12.1 \mathrm{~Hz}), 4.58-4.45(9 \mathrm{H}, \mathrm{m}), 4.45$ $(1 \mathrm{H}, \mathrm{d}, J 11.9 \mathrm{~Hz}), 4.38(1 \mathrm{H}, \mathrm{d}, J 11.9 \mathrm{~Hz}), 4.33(1 \mathrm{H}, \mathrm{d}, J 11.8 \mathrm{~Hz}), 4.29-4.22(5 \mathrm{H}$, incl. br. dd J 4.0, $9.1 \mathrm{~Hz}$ at 4.25$), 4.21-4.16(2 \mathrm{H}, \mathrm{m}), 4.13(1 \mathrm{H}$, br. dd, J 5.7, $8.2 \mathrm{~Hz}), 4.06(1 \mathrm{H}$, br. d, J $2.7 \mathrm{~Hz}), 4.01-3.96(2 \mathrm{H}, \mathrm{m})$, 3.94-3.87 (5H, m), 3.85 (1H, br. dd, J 4.8, 10.7 Hz), $3.78(1 \mathrm{H}$, br. p, J $5.1 \mathrm{~Hz}), 3.76-3.71(1 \mathrm{H}, \mathrm{m}), 3.65-3.56$ ( $3 \mathrm{H}$, incl. br. dd, J 4.8, 8.2 Hz at 3.60), $2.53(4 \mathrm{H}$, incl. sextet, J $5.4 \mathrm{~Hz}), 2.42(4 \mathrm{H}, \mathrm{t}, J 7.6 \mathrm{~Hz}), 1.68-1.11$ $(288 \mathrm{H}, \mathrm{m}), 1.06(6 \mathrm{H}, \mathrm{d}, J 6.9 \mathrm{~Hz}), 0.89(12 \mathrm{H}, \mathrm{t}, J 6.8 \mathrm{~Hz}), 0.85(9 \mathrm{H}, \mathrm{s}), 0.84(9 \mathrm{H}, \mathrm{s}), 0.71-0.61(4 \mathrm{H}, \mathrm{m}), 0.57$ $(2 \mathrm{H}, \mathrm{dt}, J 4.0,8.5 \mathrm{~Hz}), 0.03(6 \mathrm{H}, \mathrm{s}), 0.01(6 \mathrm{H}, \mathrm{s}),-0.32(2 \mathrm{H}$, br.q, $J 5.1 \mathrm{~Hz}) ; \delta_{\mathrm{C}}\left(101 \mathrm{MHz}, \mathrm{CDCl}_{3}\right): 215.2$, $174.3,174.2$, 138.5, 138.2, 137.8, 137.7, 137.6, 137.4, 137.3, 128.5, 128.45, 128.4, 128.35, 128.3, 128.2, 127.9, $127.85,127.8,127.75,127.7,127.65,127.6,127.55,127.5,106.6,106.3,105.2,88.3,88.2,87.8,83.7,83.6,81.2$, 80.2, 79.4, 78.9, 77.2, 73.4, 73.1, 72.3, 72.2, 72.0, 71.9, 71.6, 70.3, 67.0, 65.9, 63.0, 62.7, 51.6, 46.3, 41.1, 33.6, 33.5, 33.0, 31.9, 30.3, 30.2, 29.9, 29.8, 29.75, 29.7, 29.65, 29.6, 29.55, 29.5, 29.45, 29.4, 29.3, 28.7, 27.9, $27.8,27.3,27.2,27.1,25.8,24.0,23.7,22.7,22.6,18.0,16.4,15.8,14.1,10.9,-4.4,-4.5,-4.7,-4.8 ; v_{\max }$ : $3063,3031,2920,2851,1741,1714,1467,1106,836,699 \mathrm{~cm}^{-1}$.

(b) The protected furanoside (13a) $(53 \mathrm{mg}, 0.014 \mathrm{mmol})$ was dissolved in dry THF $(10 \mathrm{~mL})$ in a dry polyethylene vial equipped with an acid-proof rubber septum, followed by the addition of pyridine $(0.1 \mathrm{~mL})$ at $\mathrm{rt}$ under nitrogen. The mixture was cooled to $0{ }^{\circ} \mathrm{C}$, and then HF-pyridine complex $(70 \%$ $\mathrm{w}, 1.5 \mathrm{~mL}$ ) was added dropwise. The mixture was stirred at $43^{\circ} \mathrm{C}$ for $24 \mathrm{~h}$, then poured slowly into sat. aq. $\mathrm{NaHCO}_{3}$ and stirred until no more $\mathrm{CO}_{2}$ was liberated. The aqueous layer was re-extracted with chloroform $(3 \times 10 \mathrm{~mL})$. The combined organic layers were dried and evaporated. Column chromatography eluting with hexane/EtOAc (10:1) afforded compound (14a) as a colourless thick oil (38 mg, 76\%) [MALDI: Found $(\mathrm{M}+\mathrm{Na})^{+}: 3579.9 ; \mathrm{C}_{235} \mathrm{H}_{398} \mathrm{NaO}_{21}$ requires: 3579.9], $[\alpha]_{D}^{21}+19$ (c 1.2, $\left.\mathrm{CHCl}_{3}\right) ; \delta_{\mathrm{H}}\left(400 \mathrm{MHz}, \mathrm{CDCl}_{3}\right): 7.36-7.15(35 \mathrm{H}, \mathrm{m}), 5.16(1 \mathrm{H}$, br. s), $5.13(1 \mathrm{H}$, br. s), $5.05(1 \mathrm{H}$, br. s) 4.67 (2H, br. s), $4.55(1 \mathrm{H}, \mathrm{d}, J 11.8 \mathrm{~Hz}), 4.53-4.47(7 \mathrm{H}, \mathrm{m}), 4.45(3 \mathrm{H}, \mathrm{d}, J 11.8 \mathrm{~Hz}), 4.40(1 \mathrm{H}, \mathrm{d}, J 11.8 \mathrm{~Hz})$, 4.36-4.22 (7H, m), $4.17(1 \mathrm{H}$, br. dd, J 3.7, $6.9 \mathrm{~Hz}), 4.11(1 \mathrm{H}$, br. dd, J 3.3, $6.3 \mathrm{~Hz}), 4.07(1 \mathrm{H}$, br. d, J 2.1 $\mathrm{Hz}), 4.00-3.96(2 \mathrm{H}, \mathrm{m}), 3.94-3.88(2 \mathrm{H}, \mathrm{m}), 3.88-3.82(2 \mathrm{H}$, incl. br. dd J 4.5, 9.8Hz at 3.86), 3.81-3.75 (2H, $\mathrm{m}), 3.73(1 \mathrm{H}$, br. d, J $11.7 \mathrm{~Hz}), 3.67-3.51(5 \mathrm{H}$, incl. br. d J $5.3 \mathrm{~Hz}$ at 3.61), $2.51(2 \mathrm{H}$, sextet, J $6.7 \mathrm{~Hz})$, $2.42(6 \mathrm{H}$, incl. t J 7.2 Hz), 2.05-1.10 $(288 \mathrm{H}, \mathrm{m}), 1.06(6 \mathrm{H}, \mathrm{d}, J 6.9 \mathrm{~Hz}), 0.89(12 \mathrm{H}, \mathrm{t}, J 6.7 \mathrm{~Hz}), 0.69-0.61$ $(4 \mathrm{H}, \mathrm{m}), 0.57(2 \mathrm{H}, \mathrm{dt}, J 4.1,8.5 \mathrm{~Hz}),-0.33(2 \mathrm{H}, \mathrm{br} . \mathrm{q}, J 5.0 \mathrm{~Hz}) ; \delta_{\mathrm{C}}\left(101 \mathrm{MHz}, \mathrm{CDCl}_{3}\right): 215.2,175.1,175.0$, 138.6, 138.2, 137.6, 137.5, 137.45, 137.4, 137.2, 128.5, 128.4, 128.35, 128.3, 128.2, 128.1, 128.0, 127.95, 127.9, $127.85,127.8,127.75,127.7,127.6,127.5,106.3,106.2,105.5,88.2,88.0,87.9,83.6,80.7,80.3,79.3,79.2$, 77.2, 73.4, 72.4, 72.2, 72.1, 72.0, 71.7, 70.2, 68.0, 67.1, 65.4, 63.0, 62.9, 51.9, 51.7, 46.3, 41.1, 35.3, 35.2, 33.0, $31.9,30.3,30.2,29.8,29.7,29.65,29.6,29.55,29.5,29.45,29.4,29.3,29.2,28.7,27.5,27.4,27.3,25.7,25.6$, 23.7, 22.7, 16.4, 15.8, 14.1, 10.9; $v_{\max }: 3511,3063,3030,2918,2851,1737,1714,1467,1105,754,698 \mathrm{~cm}^{-1}$.

(c) Using the generalised procedure for debenzylation, compound (15a) was obtained as a colourless thick oil $(25 \mathrm{mg}, 86 \%)$ [MALDI: Found $(\mathrm{M}+\mathrm{Na})^{+}: 2949.7 ; \mathrm{C}_{186} \mathrm{H}_{356} \mathrm{NaO}_{21}$ requires: 2949.7], $[\alpha]_{D}^{18}+19\left(\right.$ c 2.2, $\left.\mathrm{CHCl}_{3}\right) ; \delta_{\mathrm{H}}\left(400 \mathrm{MHz}, \mathrm{CDCl}_{3}+\right.$ few drops $\left.\mathrm{CD}_{3} \mathrm{OD}\right): 5.00(1 \mathrm{H}$, br. s), $4.96(1 \mathrm{H}$, br. s), $4.90(1 \mathrm{H}$, br. s), $4.41(1 \mathrm{H}, \mathrm{dd}, J 4.2,11.6 \mathrm{~Hz}), 4.36(1 \mathrm{H}, \mathrm{dd}, J 5.1,11.9 \mathrm{~Hz}), 4.26(1 \mathrm{H}, \mathrm{dd}, J$ 11.9, $5.4 \mathrm{~Hz})$, 
$4.21(1 \mathrm{H}, \mathrm{d}, J 4.0 \mathrm{~Hz}), 4.17(1 \mathrm{H}, \mathrm{dd}, J 3.8,10.3 \mathrm{~Hz}), 4.12(1 \mathrm{H}$, br. d, J $4.8 \mathrm{~Hz}), 4.08(1 \mathrm{H}, \mathrm{br} . \mathrm{q}, J 6.6 \mathrm{~Hz})$, $4.04(1 \mathrm{H}, \mathrm{d}, J 5.1 \mathrm{~Hz}), 4.01-3.96(3 \mathrm{H}, \mathrm{m}), 3.92(1 \mathrm{H}, \mathrm{dd}, J 3.8,8.0 \mathrm{~Hz}), 3.90-3.86(2 \mathrm{H}, \mathrm{m}), 3.77(1 \mathrm{H}, \mathrm{dd}, J$ 2.7, $8.4 \mathrm{~Hz}), 3.72(1 \mathrm{H}, \mathrm{d}, J 5.1 \mathrm{~Hz}), 3.62(5 \mathrm{H}$, br. m), $3.54(1 \mathrm{H}, \mathrm{dd}, J 2.6,10.5 \mathrm{~Hz}), 2.52-2.44(2 \mathrm{H}, \mathrm{m}), 2.38$ $(6 \mathrm{H}$, incl. t, J 7.5 Hz), 1.65-1.05 (297 H, m), $1.01(6 \mathrm{H}, \mathrm{d}, J 6.9 \mathrm{~Hz}), 0.84(12 \mathrm{H}, \mathrm{t}, J 6.8 \mathrm{~Hz}), 0.66-0.57(4 \mathrm{H}$, m), $0.52(2 \mathrm{H}, \mathrm{dt}, J 4.2,8.2 \mathrm{~Hz}),-0.37(2 \mathrm{H}$, br. q, J $4.7 \mathrm{~Hz}) ; \delta_{\mathrm{C}}\left(101 \mathrm{MHz}, \mathrm{CDCl}_{3}\right): 216.2,175.05,174.9$, $109.2,108.7,81.95,81.2,79.4,78.0,77.6,77.2,76.5,72.5,71.9,65.0,63.5,63.4,61.5,52.6,35.0,32.8,31.8$, $30.1,29.6,29.55,29.5,29.4,29.3,29.25,29.2,28.9,28.6,27.3,27.2,26.1,25.3,23.5,22.6,16.1,15.7,14.0$, 10.8.; $v_{\max }: 3511,3063,3030,2918,2851,1737,1714,1467,1105,754,698 \mathrm{~cm}^{-1}$.

3.8. L-Glycerol-(1' $\rightarrow 1)-5-O-(2 R)-2-((1 R)-1-h y d r o x y-12-((2 R)-2-(14-((2 R)-2-e i c o s y l c y c l o-p r o p y l)$ tetradecyl)cyclopropyl)dodecyl)hexacosanoate- $\alpha$-D-arabinofuranosyl-(1 $\rightarrow 3)-[5-O-(2 R)-2-((1 R)-1$-hydroxy-12$((2 R)-2-(14-((2 R)-2-i c o s y l c y c l o p r o p y l) t e t r a d e c y l) c y c l o p r o p y l)-d o d e c y l) h e x a c o s a n o a t e-\alpha$-D-arabinofuranosyl$(1 \rightarrow 5)]-\alpha$-D-arabinofuranoside (15b)

(a) A solution of EDCI (48 mg, $0.25 \mathrm{mmol})$ in dry $\mathrm{CH}_{2} \mathrm{Cl}_{2}(1 \mathrm{~mL})$ was added with stirring to furanoside (9) $(28 \mathrm{mg}, 0.025 \mathrm{mmol})$, molecular sieves $4 \AA$ (50 mg), DMAP (30 mg, $0.24 \mathrm{mmol})$ and (2R)-2-((1R)-1-((tert-butyldimethylsilyl)oxy)-12-((2R)-2-(14-((2R)-2-eicosylcyclopropyl)tetradecyl) cyclopropyl)-dodecyl)hexacosanoic acid (12b) $(62 \mathrm{mg}, 0.049 \mathrm{mmol})$ [31] in dry $\mathrm{CH}_{2} \mathrm{Cl}_{2}(1 \mathrm{~mL})$ at $\mathrm{rt}$ under nitrogen. After 4 days, the precipitate was filtered and washed with $\mathrm{CH}_{2} \mathrm{Cl}_{2}(10 \mathrm{~mL})$, the solvent was evaporated and the residue was purified by column chromatography eluting with hexane/EtOAc (10:1) to afford compound (13b) as a colourless thick oil (75 mg, 84\%) [MALDI: Found (M+Na) ${ }^{+}: 3607.9$; $\mathrm{C}_{235} \mathrm{H}_{402} \mathrm{NaO}_{19} \mathrm{Si}_{2}$ requires: 3607.9$],[\alpha]_{D}^{22}+20\left(c 0.90, \mathrm{CHCl}_{3}\right)$, which showed $\delta_{\mathrm{H}}\left(400 \mathrm{MHz}, \mathrm{CDCl}_{3}\right)$ : 7.94-6.85 (35H, m), $5.16(1 \mathrm{H}$, br. s), $5.13(1 \mathrm{H}$, br. s), $5.04(1 \mathrm{H}$, br. s), $4.68(1 \mathrm{H}, \mathrm{d}, J 12.1 \mathrm{~Hz}), 4.65(1 \mathrm{H}$, d, J $12.1 \mathrm{~Hz}), 4.57-4.45(9 \mathrm{H}, \mathrm{m}), 4.45(1 \mathrm{H}, \mathrm{d}, J 11.8 \mathrm{~Hz}), 4.38(1 \mathrm{H}, \mathrm{d}, J 11.8 \mathrm{~Hz}), 4.32(1 \mathrm{H}, \mathrm{d}, J 11.8 \mathrm{~Hz})$, 4.28-4.20 (5H, m), $4.18(1 \mathrm{H}$, br. dd, J 3.6, $7.4 \mathrm{~Hz}), 4.15-4.09(1 \mathrm{H}, \mathrm{m}), 4.06(1 \mathrm{H}$, br. d, J $2.9 \mathrm{~Hz}), 4.01-3.96$ $(2 \mathrm{H}, \mathrm{m}), 3.90(3 \mathrm{H}$, br. $\mathrm{m}), 3.87-3.81(2 \mathrm{H}$, incl. br. dd, J 4.8, $10.7 \mathrm{~Hz}$ at 3.85), $3.78(1 \mathrm{H}$, br. p, J $5.1 \mathrm{~Hz})$, 3.76-3.71 $(1 \mathrm{H}, \mathrm{m}), 3.64-3.49(3 \mathrm{H}$, incl. br. dd, J 4.8, $8.3 \mathrm{~Hz}$ at 3.60), $2.60-2.47(2 \mathrm{H}, \mathrm{m}), 1.67-1.06(270 \mathrm{H}$, $\mathrm{m}), 0.89(12 \mathrm{H}, \mathrm{t}, J 6.8 \mathrm{~Hz}), 0.85(9 \mathrm{H}, \mathrm{s}), 0.84(9 \mathrm{H}, \mathrm{s}), 0.74-0.62(8 \mathrm{H}, \mathrm{m}), 0.57(4 \mathrm{H}, \mathrm{td}, J 4.1 \mathrm{~Hz}), 0.02(6 \mathrm{H}, \mathrm{s})$, $0.00(6 \mathrm{H}, \mathrm{s}),-0.33(4 \mathrm{H}$, br. q, J $4.9 \mathrm{~Hz}) ; \delta_{\mathrm{C}}\left(101 \mathrm{MHz}, \mathrm{CDCl}_{3}\right): 174.3,174.2,138.5,138.3,137.8,137.7$, 137.6, 137.4, 137.3, 128.5, 128.45, 128.4, 128.35, 128.3, 127.9, 127.85, 127.8, 127.75, 127.7, 127.65, 127.6, $127.55,127.5,106.6,106.3,105.2,88.3,88.2,87.8,83.7,83.6,81.2,80.2,79.4,78.9,77.2,73.4,73.1,72.3,72.2$, $71.9,71.6,70.3,67.0,65.9,63.0,62.7,51.6,33.6,33.5,31.9,30.3,30.2,29.9,29.8,29.7,29.6,29.5,29.4,28.7$, $27.9,27.8,27.2,27.1,25.9,25.8,24.0,22.7,18.0,15.8,14.1,11.0,10.9,-4.4,-4.5,-4.7,-4.8 ; v_{\max }: 3063$, $2925,2854,1737,1456,1101,770 \mathrm{~cm}^{-1}$.

(b) The protected glycolipid $\alpha$-D-arabinofuranoside (13b) $(70 \mathrm{mg}, 0.019 \mathrm{mmol})$ was dissolved in dry THF $(10 \mathrm{~mL})$ in a dry polyethylene vial equipped with an acid-proof rubber septum, followed by the addition of pyridine $(0.1 \mathrm{~mL})$ at $\mathrm{rt}$ under nitrogen. The mixture was cooled to $0{ }^{\circ} \mathrm{C}$, and then HF-pyridine complex $(70 \% \mathrm{w}, 1.5 \mathrm{~mL})$ was added dropwise. The mixture was stirred at $43^{\circ} \mathrm{C}$ for $24 \mathrm{~h}$, then poured slowly into sat. aq. $\mathrm{NaHCO}_{3}$ and stirred until no more $\mathrm{CO}_{2}$ was liberated. The aqueous layer was re-extracted with chloroform $(3 \times 10 \mathrm{~mL})$. The combined organic layers were dried and evaporated; column chromatography eluting with hexane/EtOAc (10:1) afforded compound (14b) as a colourless thick oil (33 mg, 51\%) [MALDI: Found (M+Na) ${ }^{+}: 3379.8 ; \mathrm{C}_{223} \mathrm{H}_{374} \mathrm{NaO}_{19}$ requires: 3379.8], $[\alpha]_{D}^{21}+22\left(c 0.90, \mathrm{CHCl}_{3}\right)$, which showed $\delta_{\mathrm{H}}\left(400 \mathrm{MHz}, \mathrm{CDCl}_{3}\right): 7.37-7.17(35 \mathrm{H}, \mathrm{m}), 5.16(1 \mathrm{H}$, br. s$)$, $5.13(1 \mathrm{H}$, br. s), $5.05(1 \mathrm{H}$, br. s), $4.67(2 \mathrm{H}$, br. s) $, 4.55(1 \mathrm{H}, \mathrm{d}, J 11.9 \mathrm{~Hz}), 4.53-4.47(8 \mathrm{H}, \mathrm{m}), 4.46(2 \mathrm{H}, \mathrm{d}, J$ $11.8 \mathrm{~Hz}), 4.41(1 \mathrm{H}, \mathrm{d}, J 11.9 \mathrm{~Hz}), 4.35-4.23(6 \mathrm{H}, \mathrm{m}), 4.17(1 \mathrm{H}$, br. dd, J 3.7, $6.9 \mathrm{~Hz}), 4.15-4.08(3 \mathrm{H}$, incl. br. t J $7.0 \mathrm{~Hz}$ at 4.12$), 4.07(1 \mathrm{H}$, br. d, J $2.1 \mathrm{~Hz}), 4.00-3.95(2 \mathrm{H}, \mathrm{m}), 3.94-3.87(2 \mathrm{H}, \mathrm{m}), 3.95-3.87(2 \mathrm{H}$, incl. br. dd, J 4.6, $10.3 \mathrm{~Hz}$ at 3.86), $3.79(1 \mathrm{H}, \mathrm{p}, J 4.8 \mathrm{~Hz}), 3.75-3.69(1 \mathrm{H}, \mathrm{m}), 3.67-3.55(3 \mathrm{H}$, incl. br. d, J $5.3 \mathrm{~Hz}$ at 3.61), 2.44-2.37 (2H, m), 1.67-1.03 (270H, m), $0.89(12 \mathrm{H}, \mathrm{t}, J 7.1 \mathrm{~Hz}), 0.69-0.61(8 \mathrm{H}, \mathrm{m}), 0.57(4 \mathrm{H}, \mathrm{dt}, J$ $4.2,8.5 \mathrm{~Hz}),-0.32(4 \mathrm{H}$, br. q, J $4.9 \mathrm{~Hz}) ; \delta_{\mathrm{C}}\left(101 \mathrm{MHz}, \mathrm{CDCl}_{3}\right): 175.1,175.0,138.5,138.2,137.6,137.5$, 137.4, 137.4, 137.2, 128.5, 128.4, 128.35, 128.3, 128.0, 127.95, 127.9, 127.85, 127.8, 127.75, 127.7, 127.6, $127.5,106.3,106.2,105.5,88.2,88.0,87.9,83.6,80.7,80.3,79.3,79.2,77.2,73.4,72.4,72.2,72.0,71.9,71.7$, $70.3,67.1,65.4,63.1,63.0,62.0,51.9,51.8,35.3,35.2,34.2,31.9,30.3,30.2,29.8,29.75,29.7,29.65,29.6$, 
$29.5,29.4,28.7,27.5,27.4,25.8,25.7,24.9,22.7,22.6,20.4,15.8,14.1,10.9 ; v_{\max }: 3511,3062,2921,2854$, $1733,1725,1456,1115,754 \mathrm{~cm}^{-1}$.

(c) Using the generalised debenzylation procedure, compound $(\mathbf{1 5 b})$ was obtained as a colourless thick oil (22 mg, 88\%) [MALDI: Found $(\mathrm{M}+\mathrm{Na})^{+}: 2749.5 ; \mathrm{C}_{174} \mathrm{H}_{332} \mathrm{NaO}_{19}$ requires: 2749.5$],[\alpha]_{D}^{18}+18(c$ 2.2, $\left.\mathrm{CHCl}_{3}\right)$, which showed $\delta_{\mathrm{H}}\left(400 \mathrm{MHz}, \mathrm{CDCl}_{3}+\right.$ few drops $\left.\mathrm{CD}_{3} \mathrm{OD}\right): 5.00(1 \mathrm{H}$, br. s), $4.96(1 \mathrm{H}$, br. s), $4.90(1 \mathrm{H}$, br. s), $4.41(1 \mathrm{H}, \mathrm{dd}, J$ 4.6, $11.7 \mathrm{~Hz}), 4.37(1 \mathrm{H}$, br. dd, J 5.1, $11.9 \mathrm{~Hz}), 4.26(1 \mathrm{H}, \mathrm{dd}, J 5.0,11.7 \mathrm{~Hz})$, $4.20(1 \mathrm{H}, \mathrm{dd}, J 4.7,11.8 \mathrm{~Hz}), 4.16(1 \mathrm{H}$, br. q, J $4.8 \mathrm{~Hz}), 4.12(1 \mathrm{H}$, br. d, J $6.2 \mathrm{~Hz}), 4.10-4.06(2 \mathrm{H}$, incl. br. t, $J 7.4 \mathrm{~Hz}$ at 4.08$), 4.05(1 \mathrm{H}$, br. $\mathrm{d}, J 5.6 \mathrm{~Hz}), 4.02-3.96(3 \mathrm{H}$, incl. br. d, J $8.5 \mathrm{~Hz}$ at 3.99$), 3.95-3.86(3 \mathrm{H}, \mathrm{m})$, $3.77(1 \mathrm{H}, \mathrm{dd}, J 4.2,7.4 \mathrm{~Hz}), 3.72(1 \mathrm{H}, \mathrm{d}, J 5.4 \mathrm{~Hz}), 3.67-3.57(4 \mathrm{H}$, incl. br. d, J $10.5 \mathrm{~Hz}$ at 3.63), $3.54(1 \mathrm{H}$, $\mathrm{dd}, J 2.6,9.7 \mathrm{~Hz}), 2.42-2.35(2 \mathrm{H}, \mathrm{m}), 1.64-1.04(277 \mathrm{H}, \mathrm{m}), 0.84(12 \mathrm{H}, \mathrm{t}, J 6.7 \mathrm{~Hz}), 0.64-0.56(8 \mathrm{H}, \mathrm{m}), 0.52$ $(4 \mathrm{H}, \mathrm{dt}, J 4.1,8.4 \mathrm{~Hz}),-0.37(4 \mathrm{H}$, br. q, J $4.9 \mathrm{~Hz}) ; \delta_{\mathrm{C}}\left(101 \mathrm{MHz}, \mathrm{CDCl}_{3}+\right.$ few drops $\left.\mathrm{CD}_{3} \mathrm{OD}\right): 175.0,174.9$, 108.7, 108.0, 81.7, 81.3, 79.7, 79.4, 77.9, 77.5, 77.2, 76.3, 72.4, 71.9, 69.7, 69.15, 68.9, 67.6, 65.1, 63.4, 62.2, $61.4,57.8,52.6,34.9,31.8,30.1,29.55,29.5,29.45,29.4,29.3,29.2,29.15,29.1,28.6,27.3,25.3,22.5,20.5$, 15.6, 13.9, 10.7.; $v_{\max }: 3399,2920,2851,1734,1467,1043 \mathrm{~cm}^{-1}$.

3.9. L-Glycerol-(1' $\rightarrow 1)-5-O-(2 R)-2-((1 R)-1-h y d r o x y-17-((1 S, 2 R)-2-((22 S)-22-m e t h y l-21-o x o t e t r a c o n t a n-2-y l)$ cyclopropyl)heptadecyl)hexacosanoate- $\alpha$-D-arabinofuranosyl- $(1 \rightarrow 3)-[-5-O-(2 R)-2-((1 R)-1-h y d r o x y-17-((1 S, 2 R)$ -2-((22S)-22-methyl-21-oxotetracontan-2-yl)cyclo-propyl)heptadecyl)hexacosanoate- $\alpha$-D-arabinofuranosyl$(1 \rightarrow 5)]-\alpha$-D-arabinofuranoside (15c)

(a) A solution of EDCI ( $35 \mathrm{mg}, 0.18 \mathrm{mmol})$ in dry $\mathrm{CH}_{2} \mathrm{Cl}_{2}(1 \mathrm{~mL})$ was added to a stirred solution of $\alpha$-D-arabinofuranoside (9) (22 mg, $0.019 \mathrm{mmol})$, molecular sieves $4 \AA$ ( $25 \mathrm{mg})$, DMAP ( $23 \mathrm{mg}, 0.18 \mathrm{mmol})$ and (2R)-2-((1R)-1-((tert-butyldimethylsilyl)oxy)-17-((1S,2R)-2-((22S)-22-methyl-21-oxotetra-contan-2yl) cyclopropyl)-heptadecyl)hexacosanoic acid (12c) $(50 \mathrm{mg}, 0.035 \mathrm{mmol})$ [32] in dry $\mathrm{CH}_{2} \mathrm{Cl}_{2}(1 \mathrm{~mL})$ at $\mathrm{rt}$ under nitrogen. The mixture was stirred for 4 days, then the precipitate was filtered off and washed with $\mathrm{CH}_{2} \mathrm{Cl}_{2}(10 \mathrm{~mL})$, the solvent was evaporated and the residue was purified by column chromatography eluting with hexane/EtOAc (10:1) to afford the title compound (13c) as a colourless thick oil (35 mg, $46 \%$ ) [MALDI: Found $(\mathrm{M}+\mathrm{Na})^{+}: 3892.2 ; \mathrm{C}_{253} \mathrm{H}_{438} \mathrm{NaO}_{21} \mathrm{Si}_{2}$ requires: 3892.2], $[\alpha]_{D}^{20}+14\left(c\right.$ 3.0, $\left.\mathrm{CHCl}_{3}\right)$, which showed $\delta_{\mathrm{H}}\left(400 \mathrm{MHz}, \mathrm{CDCl}_{3}\right): 7.54-7.00(35 \mathrm{H}, \mathrm{m}), 5.16(1 \mathrm{H}$, br. s), $5.13(1 \mathrm{H}, \mathrm{br} . \mathrm{s}), 5.04(1 \mathrm{H}, \mathrm{br}$. s), $4.68(1 \mathrm{H}, \mathrm{d}, J 12.1 \mathrm{~Hz}), 4.65(1 \mathrm{H}, \mathrm{d}, J 12.1 \mathrm{~Hz}), 4.58-4.47(9 \mathrm{H}, \mathrm{m}), 4.46(1 \mathrm{H}, \mathrm{d}, J 11.9 \mathrm{~Hz}), 4.39(1 \mathrm{H}, \mathrm{d}, J$ $11.8 \mathrm{~Hz}), 4.33(1 \mathrm{H}, \mathrm{d}, J 11.9 \mathrm{~Hz}), 4.30-4.22(5 \mathrm{H}$, incl. br. dd, J 3.9, $10.4 \mathrm{~Hz}$ at 4.26$), 4.22-4.16(2 \mathrm{H}, \mathrm{m}), 4.13$ $(1 \mathrm{H}$, br. dd, J 5.6, $8.4 \mathrm{~Hz}), 4.06(1 \mathrm{H}$, br. d, J $2.9 \mathrm{~Hz}), 4.01-3.97(2 \mathrm{H}, \mathrm{m}), 3.95-3.87(5 \mathrm{H}, \mathrm{m}), 3.85(1 \mathrm{H}, \mathrm{br}$. dd, J 3.8, 9.4 Hz), $3.78(1 \mathrm{H}$, br. p, J $4.9 \mathrm{~Hz}), 3.76-3.72(1 \mathrm{H}, \mathrm{m}), 3.65-3.55(3 \mathrm{H}$, incl. br. dd J 4.8, $8.3 \mathrm{~Hz}$ at 3.60), $2.53(4 \mathrm{H}$, incl. sextet, J 5.6 Hz), $2.42(4 \mathrm{H}, \mathrm{t}, J 7.5 \mathrm{~Hz}), 1.62-1.14(292 \mathrm{H}, \mathrm{m}), 1.06(6 \mathrm{H}, \mathrm{d}, J 6.9 \mathrm{~Hz})$, $0.89(12 \mathrm{H}, \mathrm{t}, J 6.8 \mathrm{~Hz}), 0.88(6 \mathrm{H}, \mathrm{d}, J 6.8 \mathrm{~Hz}), 0.85(9 \mathrm{H}, \mathrm{s}), 0.84(9 \mathrm{H}, \mathrm{s}), 0.75-0.62(2 \mathrm{H}, \mathrm{m}), 0.51-0.40(2 \mathrm{H}$, $\mathrm{m}), 0.24-0.08(6 \mathrm{H}, \mathrm{m}), 0.03(6 \mathrm{H}, \mathrm{s}), 0.01(6 \mathrm{H}, \mathrm{s}) ; \delta_{\mathrm{C}}\left(101 \mathrm{MHz}, \mathrm{CDCl}_{3}\right): 215.1,174.2,174.1,138.6,138.3$, $137.8,137.7,137.6,137.55,137.5,128.45,128.4,128.35,128.3,128.25,128.2,127.9,127.85,127.8,127.75$, 127.7, 127.65, 127.6, 127.55, 127.5, 106.6, 106.3, 105.2, 88.3, 88.2, 87.8, 83.8, 83.7, 81.2, 80.2, 79.5, 78.9, 77.2, 73.4, 73.2, 72.3, 72.2, 72.0, 71.9, 71.6, 70.3, 67.0, 65.9, 63.0, 62.7, 51.6, 46.3, 41.1, 38.1, 37.4, 34.5, 33.7, 33.6, 33.0, 31.9, 30.1, 29.9, 29.8, 29.75, 29.7, 29.65, 29.6, 29.55, 29.5, 29.45, 29.4, 29.35, 29.3, 27.8, 27.4, 27.3, 27.2, 27.1, 26.1, 25.9, 25.8, 24.1, 23.7, 22.7, 22.6, 19.7, 18.6, 18.0, 16.4, 14.1, 10.5, -4.4, -4.5, -4.7, -4.8; $v_{\max }$ : $3063,3032,2919,2851,1739,1714,1467,1105,836,698 \mathrm{~cm}^{-1}$.

(b) The protected glycolipid furanoside $(\mathbf{1 3 c})(31 \mathrm{mg}, 0.0080 \mathrm{mmol})$ was dissolved in dry THF $(10 \mathrm{~mL})$ in a dry polyethylene vial equipped with an acid-proof rubber septum, followed by the addition of pyridine $(0.1 \mathrm{~mL})$ at $\mathrm{rt}$ under nitrogen. The mixture was cooled to $0{ }^{\circ} \mathrm{C}$, and then HF-pyridine complex as $(70 \% \mathrm{w}, 1.5 \mathrm{~mL})$ was added dropwise. The mixture was stirred at $43^{\circ} \mathrm{C}$ for $24 \mathrm{~h}$, then poured slowly into sat. aq. $\mathrm{NaHCO}_{3}$ and stirred until no more $\mathrm{CO}_{2}$ was liberated. The aqueous layer was re-extracted with chloroform $(3 \times 10 \mathrm{~mL})$. The combined organic layers were dried and evaporated; column chromatography eluting with hexane/EtOAc (10:1) afforded compound (14c) as a colourless thick oil $\left(19 \mathrm{mg}, 66 \%\right.$ ) [MALDI: Found $(\mathrm{M}+\mathrm{Na})^{+}: 3664.1 ; \mathrm{C}_{241} \mathrm{H}_{410} \mathrm{NaO}_{21}$ requires: 3664.1$],[\alpha]_{D}^{18}+25$ (c 1.1, $\left.\mathrm{CHCl}_{3}\right)$, which showed $\delta_{\mathrm{H}}\left(400 \mathrm{MHz}, \mathrm{CDCl}_{3}\right): 7.39-7.18(35 \mathrm{H}, \mathrm{m}), 5.16(1 \mathrm{H}$, br. s), $5.13(1 \mathrm{H}$, br. s), $5.04(1 \mathrm{H}$, br. s), $4.67(2 \mathrm{H}$, br. s), $4.55(1 \mathrm{H}, \mathrm{d}, J 11.9 \mathrm{~Hz}), 4.53-4.47(8 \mathrm{H}, \mathrm{m}), 4.45(2 \mathrm{H}, \mathrm{d}, J 11.8 \mathrm{~Hz}), 4.40$ 
$(1 \mathrm{H}, \mathrm{d}, J 11.8 \mathrm{~Hz}), 4.29$ (6H, br. m), $4.17(1 \mathrm{H}$, br. dd, J 3.8, $6.9 \mathrm{~Hz}), 4.11(1 \mathrm{H}$, br. dd, J 3.4, 6.2 Hz), 4.07 $(1 \mathrm{H}$, br. d, J $2.5 \mathrm{~Hz}), 3.99-3.95(2 \mathrm{H}, \mathrm{m}), 3.94-3.87(2 \mathrm{H}, \mathrm{m}), 3.87-3.82(2 \mathrm{H}$, incl. br. dd, J 4.6, $10.3 \mathrm{~Hz}$ at 3.85), $3.79(1 \mathrm{H}$, br. p, J $4.9 \mathrm{~Hz}), 3.72(1 \mathrm{H}$, br.dd, J 1.7, $11.6 \mathrm{~Hz}), 3.64-3.57(5 \mathrm{H}, \mathrm{m}), 2.51(2 \mathrm{H}$, sextet, $J$ $6.8 \mathrm{~Hz}), 2.42(6 \mathrm{H}$, incl. t, J 7.5 Hz), $1.87-1.08(300 \mathrm{H}, \mathrm{m}), 1.05(6 \mathrm{H}, \mathrm{d}, J 6.9 \mathrm{~Hz}), 0.89(12 \mathrm{H}, \mathrm{t}, J 7.3 \mathrm{~Hz})$, $0.72-0.62(2 \mathrm{H}, \mathrm{m}), 0.50-0.38(2 \mathrm{H}, \mathrm{m}), 0.25-0.05(6 \mathrm{H}, \mathrm{m}) ; \delta_{\mathrm{C}}\left(101 \mathrm{MHz}, \mathrm{CDCl}_{3}\right): 215.2,175.1,175.0,138.6$, $138.3,137.7,137.5,137.45,137.4,137.2,128.5,128.4,128.35,128.3,128.2,128.0,127.95,127.9,127.85$, 127.8, 127.75, 127.7, 127.6, 106.3, 106.2, 105.4, 88.2, 88.0, 87.9, 83.6, 80.7, 80.3, 79.3, 79.2, 77.2, 73.4, 72.4, 72.2, 72.1, 72.0, 71.9, 71.7, 70.3, 67.1, 65.4, 63.0, 62.9, 51.9, 51.7, 46.3, 41.1, 38.1, 37.4, 35.3, 35.2, 34.5, 33.0, $31.9,30.1,29.75,29.7,29.65,29.6,29.55,29.5,29.45,29.4,29.3,27.5,27.4,27.35,27.3,26.1,25.7,23.7,22.7$, $19.7,18.6,16.4,14.1,10.5 ; v_{\max }: 3457,3063,3031,2919,2852,1737,1715,1464,1104,734,698 \mathrm{~cm}^{-1}$.

(c) Using the generalised debenzylation procedure, compound (15c) was obtained as a colourless oil $(9.0 \mathrm{mg}, 82 \%)$ [MALDI: Found $(\mathrm{M}+\mathrm{Na})^{+}: 3033.8 ; \mathrm{C}_{192} \mathrm{H}_{368} \mathrm{NaO}_{21}$ requires: 3033.8$],[\alpha]_{D}^{18}+26(c 0.90$, $\left.\mathrm{CHCl}_{3}\right) ; \delta_{\mathrm{H}}\left(400 \mathrm{MHz}, \mathrm{CDCl}_{3}+\right.$ few drops $\left.\mathrm{CD}_{3} \mathrm{OD}\right): 5.01(1 \mathrm{H}$, br. s), $4.96(1 \mathrm{H}$, br. s), $4.91(1 \mathrm{H}$, br. s), 4.42 $(1 \mathrm{H}, \mathrm{dd}, J$ J 4.9, $12.1 \mathrm{~Hz}), 4.37(1 \mathrm{H}, \mathrm{dd}, J 4.6,11.8 \mathrm{~Hz}), 4.27(1 \mathrm{H}, \mathrm{dd}, J 4.7,11.1 \mathrm{~Hz}), 4.22(1 \mathrm{H}, \mathrm{br} . \mathrm{d}, J 4.7$ $\mathrm{Hz}), 4.18(1 \mathrm{H}, \mathrm{dd}, J 4.2,9.0 \mathrm{~Hz}), 4.13(1 \mathrm{H}$, br. q, J $5.4 \mathrm{~Hz}), 4.09(1 \mathrm{H}, \mathrm{t}, J 7.0 \mathrm{~Hz}), 4.06(1 \mathrm{H}, \mathrm{d}, J 5.1 \mathrm{~Hz})$, 4.02-3.97 (3H, br. m), $3.93(1 \mathrm{H}$, br. dd, J 3.3, $7.3 \mathrm{~Hz}), 3.89(2 \mathrm{H}$, br. m), 3.80-3.72 $(2 \mathrm{H}, \mathrm{m}), 3.67-3.58(5 \mathrm{H}$, m), $3.55(1 \mathrm{H}, \mathrm{dd}, J 2.4,9.6 \mathrm{~Hz}), 2.53-2.44(2 \mathrm{H}$, sextet, $J 6.6 \mathrm{~Hz}), 2.39(6 \mathrm{H}$, incl. t, J 7.4 Hz), 1.57-1.06 $(210 \mathrm{H}, \mathrm{m}), 1.01(6 \mathrm{H}, \mathrm{d}, J 6.9 \mathrm{~Hz}), 0.85(12 \mathrm{H}, \mathrm{t}, J 7.5 \mathrm{~Hz}), 0.67-0.57(2 \mathrm{H}, \mathrm{m}), 0.47-0.34(2 \mathrm{H}, \mathrm{m}), 0.21-0.02$ $(6 \mathrm{H}, \mathrm{m}) ; \delta_{\mathrm{C}}\left(101 \mathrm{MHz}, \mathrm{CDCl}_{3}\right): 215.8,175.0,174.75,109.3,108.7,87.1,82.95,82.9,80.9,79.4,78.8,78.2$, 77.9, 77.2, 72.6, 71.7, 68.8, 65.0, 64.0, 63.3, 61.8, 52.5, 46.3, 41.1, 38.1, 37.4, 35.1, 34.4, 33.0, 32.9, 31.9, 30.0, $29.7,29.6,29.55,29.5,29.45,29.4,29.35,29.3,27.4,27.35,27.3,27.25,27.2,26.1,25.45,25.4,23.6,23.55$, 22.6, 19.6, 18.6, 16.3, 16.2, 14.1, 10.4.; $v_{\max }: 3434,2919,2852,1736,1717,1467,1104,735,699 \mathrm{~cm}^{-1}$.

Supplementary Materials: Supplementary data related to this article, including the Figures S1-S7 and Table S1 referred to in the text, can be found online at http://www.mdpi.com/1420-3049/24/19/3596/s1.

Author Contributions: Conceptualisation, J.R.A.D. and M.S.B.; methodology, O.T.A. and M.O.M.; analysis, O.T.A. and J.R.A.; investigation, O.T.A.; supervision, J.R.A.D. and M.S.B.; resources, M.S.B.; original draft preparation, O.T.A. and J.R.A.D.; writing-review and editing, M.O.M. and M.S.B.; project administration, J.R.A.D. and M.S.B.

Funding: This research received no external funding other than the $\mathrm{PhD}$ studentships listed in the Acknowledgements.

Acknowledgments: We thank the Iraqi Ministry of Higher Education and Scientific Research and Mosul University for the award of a PhD studentship to O.T.A. and the Ministry of Higher Education and Scientific Research, Kurdistan Region, Iraq, for the award of a PhD grant to M.O.M. We thank the EPSRC National Mass Spectrometry Service Centre, Swansea, for MALDI measurements.

Conflicts of Interest: There are no conflicts of interest for any of the authors of this paper.

\section{References}

1. Marrakchi, H.; Lanéelle, M.A.; Daffé, M. Mycolic acids: Structures biosynthesis and beyond. Chem. Biol. 2014, 21, 67-85. [CrossRef] [PubMed]

2. Verschoor, J.A.; Baird, M.S.; Grooten, J. Towards understanding the functional diversity of cell wall mycolic acids of Mycobacterium tuberculosis. Prog. Lipid Res. 2012, 51, 325-339. [CrossRef]

3. Uenishi, Y.; Kusunose, N.; Yano, I.; Sunagawa, M. Isolation and Identification of Arabinose Mycolates of Cell Wall Skeleton (CWS) Derived from Mycobacterium Bovis BCG Tokyo 172 (SMP-105). J. Microbiol. Methods 2010, 80, 302-305. [CrossRef] [PubMed]

4. Miyauchi, M.; Murata, M.; Shibuya, K.; Koga-Yamakawa, E.; Uenishi, Y.; Kusunose, N.; Sunagawa, M.; Yano, I.; Kashiwazaki, Y. Arabino-Mycolates Derived from Cell-Wall Skeleton of Mycobacterium Bovis BCG as a Prominent Structure for Recognition by Host Immunity. Drug Discov. Ther. 2011, 5, 130-135. [CrossRef]

5. Kleinnijenhuis, J.; Oosting, M.; Joosten, L.A.B.; Netea, M.G.; Van Crevel, R. Innate Immune Recognition of Mycobacterium tuberculosis. Clin. Dev. Immunol. 2011, 1-12. [CrossRef] [PubMed]

6. Liu, C.; Richards, M.R.; Lowary, T.L. Probing the Effect of Acylation on Arabinofuranose Ring Conformation in Di- and Trisaccharide Fragments of Mycobacterial Arabinogalactan. J. Org. Chem. 2010, 75, 4992-5007. [CrossRef] [PubMed] 
7. Ayers, J.D.; Lowary, T.L.; Morehouse, C.B.; Besra, G.S. Synthetic arabinofuranosyl oligosaccharides as Mycobacterial arabinosyltransferase substrates. Bioorg. Med. Chem. Lett. 1998, 8, 437-442. [CrossRef]

8. Mereyala, H.B.; Hotha, S.; Gurjar, M.K. Synthesis of pentaarabinofuranosyl structure motif A of Mycobacterium tuberculosis. Chem. Commun. 1998, 685-686. [CrossRef]

9. Backus, K.M.; Dolan, M.A.; Barry, C.S.; Joe, M.; McPhie, P.; Boshoff, H.I.M.; Lowary, T.L.; Davis, B.; Barry, C.E. The Three Mycobacterium tuberculosis Antigen 85 Isoforms Have Unique Substrates and Activities Determined by Non-active Site Regions. J. Biol. Chem. 2014, 289, 25041-25053. [CrossRef]

10. Ishiwata, A.; Akao, H.; Ito, Y.; Sunagawa, M.; Kusunose, N.; Kashiwazaki, Y. Synthesis and TNF-alpha inducing activities of mycoloyl-arabinan motif of mycobacterial cell wall components. Bioorg. Med. Chem. 2006, 14, 3049-3061. [CrossRef]

11. Sunakawa, J.; Uenishi, Y.; Kususe, N.; Ito, Y.; Ishiwata, A.; Arao, H.; Yan, I.; Fujita, Y. New Arabino-Mycolate Compound. JP2006312604, 16 September 2006.

12. Kremer, L.; de Chastellier, C.; Dobson, G.; Gibson, K.J.C.; Bifani, P.; Balor, S.; Gorvel, J.P.; Locht, C.; Minnikin, D.E.; Besra, G.S. Identification and structural characterization of an unusual mycobacterial monomeromycoloyl-diacylglycerol. Mol. Microbiol. 2005, 57, 1113-1126. [CrossRef] [PubMed]

13. Andersen, C.S.; Agger, E.M.; Rosenkrands, I.; Gomes, J.M.; Bhowruth, V.; Gibson, K.J.C.; Petersen, R.V.; Minnikin, D.E.; Besra, G.S.; Andersen, P.A. Simple Mycobacterial Monomycolated Glycerol Lipid Has Potent Immunostimulatory Activity. J. Immunol. 2009, 182, 424-432. [CrossRef] [PubMed]

14. Andersen, C.S.; Rosenkrands, I.; Olsen, A.W.; Nordly, P.; Christensen, D.; Lang, R.; Kirschning, C.; Gomes, J.M.; Bhowruth, V.; Minnikin, D.E.; et al. Novel Generation Mycobacterial Adjuvant Based on Liposome-Encapsulated Monomycoloyl Glycerol from Mycobacterium bovis Bacillus Calmette-Guérin. J. Immunol. 2009, 183, 2294-2302. [CrossRef] [PubMed]

15. Bhowruth, V.; Minnikin, D.E.; Agger, E.M.; Andersen, P.; Bramwell, V.W.; Perrie, Y.; Besra, G.S. Adjuvant properties of a simplified C32 monomycoloylglycerol analogue. Bioorg. Med. Chem. Lett. 1999, 19, 2029-2032. [CrossRef] [PubMed]

16. Hattori, Y.; Matsunaga, I.; Komori, T.; Urakawa, T.; Nakamura, T.; Fujiwara, N.; Hiromatsu, K.; Harashima, H.; Sugita, M. Glycerol monomycolate, a latent tuberculosis-associated mycobacterial lipid, induces eosinophilic hypersensitivity responses in guinea pigs. Biochem. Biophys. Res. Commun. 2011, 409, 304-307. [CrossRef] [PubMed]

17. Hattori, Y.; Morita, D.; Fujiwara, N.; Mori, D.; Nakamura, T.; Harashima, H.; Yamasaki, S.; Sugita, M. Glycerol monomycolate is a novel ligand for the human, but not mouse macrophage inducible C-type lectin, Mincle. J. Biol. Chem. 2014, 289, 15405-15412. [CrossRef] [PubMed]

18. Watanabe, M.; Ohta, A.; Sasaki, S.; David, E.; Minnikin, D.E. Structure of a New Glycolipid from the Mycobacterium avium-Mycobacterium intracellulare Complex. J. Bacteriol. 1999, 181, 2293-2297. [PubMed]

19. Mohammed, M.O.; Baird, M.S.; Al Dulayymi, J.R. Mycolyl arabino glycerols from synthetic mycolic acids. Tetrahedron Lett. 2015, 56, 3268-3272. [CrossRef]

20. Watanabe, M.; Kudoh, S.; Yamada, Y.; Iguchi, K.; Minnikin, D.E. A new glycolipid from Mycobacterium avium-Mycobacterium intracellulare complex. Biochim. Biophys. Acta 1992, 1165, 53-60. [CrossRef]

21. Honda, I.; Kawajiri, K.; Watanabe, M.; Tioda, I.; Kawamata, K.; Minnikin, D.E. Evaluation of the use of 5-mycoloyl- $\beta$-arabinofuranosyl-( $1 \rightarrow 2)$-5-mycoloyl- $\alpha$-arabinofuranosyl-( $\left.1 \rightarrow 1^{\prime}\right)$-glycerol in serodiagnosis of Mycobacterium avium-intracellulare complex infection. Res. Microbiol. 1993, 144, 229-235. [CrossRef]

22. Rombouts, Y.; Brust, B.; Ojha, A.K.; Maes, E.; Coddeville, B.; Elass-Rochard, E.; Kremer, L.; Guerardel, Y. Mycobacteria to Cell Wall-inhibitory Drugs Decreases Production of Arabinoglycerolipid Related to Mycolyl-arabinogalactan-peptidoglycan Metabolism. J. Biol. Chem. 2012, 287, 11060-11069. [CrossRef] [PubMed]

23. Elass-Rochard, E.; Rombouts, Y.; Coddeville, B.; Maes, E.; Blervaque, R.; Hot, D.; Kremer, L.; Guérardel, Y. Structural Determination and Toll-like Receptor 2-dependent Proinflammatory Activity of Dimycolyl-diarabino-glycerol from Mycobacterium marinum. J. Biol. Chem. 2012, 287, 34432-34444. [CrossRef] [PubMed]

24. McNeil, M.; Daffe, M.; Brennan, P. Location of the mycolyl ester substituents in the cell walls of mycobacteria. J. Am. Soc. Biochem. Mol. Biol. 1991, 266, 13217-13223. 
25. Mohammed, M.O.; Al Dulayymi, J.R.; Baird, M.S. Preparation of the tri-arabino di-mycolate fragment of mycobacterial arabinogalactan from defined synthetic mycolic acids. Carbohydrate Res. 2017, 437, $36-42$. [CrossRef] [PubMed]

26. Ali, O.T.; Mohammed, M.O.; Gates, P.J.; Baird, M.S.; Al Dulayymi, J.R. The synthesis of mycobacterial dimycoloyl diarabinoglycerol based on defined synthetic mycolic acids. Chem. Phys. Lipids 2019, 221, 207-218. [CrossRef] [PubMed]

27. Chancellor, A.; Tocheva, A.S.; Cave-Ayland, C.; Tezera, L.; White, A.; Al Dulayymi, J.R.; Bridgeman, J.S.; Tews, I.; Wilson, S.; Lissin, N.M.; et al. CD1b-restricted GEM T cell responses are modulated by Mycobacterium tuberculosis mycolic acid meromycolate chains. PNAS 2017, 114, E10956-E10964. [CrossRef]

28. Tima, H.G.; Al Dulayymi, J.R.; Denis, O.; Lehebel, P.; Baols, K.S.; Mohammed, M.O.; L'Homme, L.; Sahb, M.M.; Potemberg, G.; Legrand, S.; et al. Inflammatory Properties and Adjuvant Potential of Synthetic Glycolipids Homologous to Mycolate Esters of the Cell Wall of Mycobacterium tuberculosis. J. Innate Immunity 2016, 9, 162-180. [CrossRef]

29. Jones, A.; Pitts, M.; Al Dulayymi, J.R.; Gibbons, J.; Ramsay, A.; Goletti, D.; Gwenin, C.D.; Baird, M.S. New synthetic lipid antigens for rapid serological diagnosis of tuberculosis. PLoS ONE 2017, 12, e0181414. [CrossRef]

30. Koza, G.; Theunissen, C.; Al-Dulayymi, J.R.; Baird, M.S. The synthesis of single enantiomers of mycobacterial ketomycolic acids containing cis-cyclopropanes. Tetrahedron 2009, 65, 10214-10229. [CrossRef]

31. Al Dulayymi, J.R.; Baird, M.S.; Roberts, E. The synthesis of a single enantiomer of a major $\alpha$-mycolic acid of M. tuberculosis. Tetrahedron 2005, 61, 11939-11951. [CrossRef]

32. Koza, G.; Muzael, M.; Schubert-Rowles, R.R.; Theunissen, C.; Al Dulayymi, J.R.; Baird, M.S. The synthesis of methoxy and keto mycolic acids containing methyl-trans-cyclopropanes. Tetrahedron 2013, 69, 6285-6296. [CrossRef]

Sample availability: Samples of the final materials are available from the authors.

(C) 2019 by the authors. Licensee MDPI, Basel, Switzerland. This article is an open access article distributed under the terms and conditions of the Creative Commons Attribution (CC BY) license (http://creativecommons.org/licenses/by/4.0/). 